\title{
Galaxy mergers in EAGLE do not induce a significant amount of black hole growth yet do increase the rate of luminous AGN
}

\author{
Stuart McAlpine ${ }^{\ominus},{ }^{1 \star}$ Chris M. Harrison ${ }^{\oplus},{ }^{2}$ David J. Rosario, ${ }^{3}$ David M. Alexander, ${ }^{3}$ \\ Sara L. Ellison ${ }^{\oplus},{ }^{4}$ Peter H. Johansson ${ }^{1}$ and David R. Patton ${ }^{\oplus 5}$ \\ ${ }^{1}$ Department of Physics, University of Helsinki, Gustaf Hällströmin katu 2 P.O. Box 64, FI-00014 Helsinki, Finland \\ ${ }^{2}$ School of Mathematics, Statistics and Physics, Newcastle University, Newcastle upon Tyne NE1 7RU, UK \\ ${ }^{3}$ Centre for Extragalactic Astronomy, Department of Physics, Durham University, South Road, Durham DH1 3LE, UK \\ ${ }^{4}$ Department of Physics and Astronomy, University of Victoria, Finnerty Road, Victoria, British Columbia V8P 1A1, Canada \\ ${ }^{5}$ Department of Physics and Astronomy, Trent University, 1600 West Bank Drive, Peterborough, Ontario K9L 0G2, Canada
}

Accepted 2020 April 21. Received 2020 April 20; in original form 2020 January 31

\begin{abstract}
We investigate the connection between galaxy-galaxy mergers and enhanced black hole $(\mathrm{BH})$ growth using the cosmological hydrodynamical EAGLE simulation. We do this via three methods of analysis, investigating: the merger fraction of AGN, the AGN fraction of merging systems, and the AGN fraction of galaxies with close companions. In each case, we find an increased abundance of AGN within merging systems relative to control samples of inactive or isolated galaxies (by up to a factor of $\approx 3$ depending on the analysis method used), confirming that mergers are enhancing $\mathrm{BH}$ accretion rates for at least a subset of the galaxy population. The greatest excess of AGN triggered via a merger are found in lower mass $\left(M_{*} \sim 10^{10} \mathrm{M}_{\odot}\right)$ gas rich $\left(f_{\text {gas }}>0.2\right)$ central galaxies with lower mass BHs $\left(M_{\mathrm{BH}} \sim 10^{7} \mathrm{M}_{\odot}\right)$ at lower redshifts $(z<1)$. We find no enhancement of AGN triggered via mergers in more massive galaxies $\left(M_{*} \gtrsim 10^{11} \mathrm{M}_{\odot}\right)$. The enhancement of AGN is not uniform throughout the phases of a merger, and instead peaks within the early remnants of merging systems (typically lagging $\approx 300 \mathrm{Myr}$ post-coalescence of the two galaxies at $z=0.5)$. We argue that neither major $\left(M_{*, 1} / M_{*, 2} \geq \frac{1}{4}\right)$ nor minor mergers $\left(\frac{1}{10}<M_{*, 1} / M_{*, 2}<\frac{1}{4}\right)$ are statistically relevant for enhancing $\mathrm{BH}$ masses globally. Whilst at all redshifts the galaxies experiencing a merger have accretion rates that are on average 2-3 times that of isolated galaxies, the majority of mass that is accreted on to BHs occurs outside the periods of a merger. We compute that on average no more than 15 percent of a BHs final day mass comes from the enhanced accretion rates triggered via a merger.
\end{abstract}

Key words: galaxies: active-galaxies: evolution-galaxies: formation-galaxies: highredshift-galaxies: interactions.

\section{INTRODUCTION}

The physical link between actively accreting supermassive black holes (BHs, referred to as active galactic nuclei, or AGNs) and galaxy-galaxy interactions is the subject of a complex and ongoing debate, first systematically explored over $30 \mathrm{yr}$ ago (Sanders et al. 1988). Theoretically, there are compelling reasons why one would expect such a link to exist. For example, the strong gravitational torques induced during gas-rich major mergers (typically defined as $\leq 4: 1$ stellar mass ratios) can effectively funnel gas towards the nuclei, fuelling bursts of star formation and nuclear activity (e.g. Barnes \& Hernquist 1991; Mihos \& Hernquist 1996; Blumenthal \& Barnes 2018). Additionally, numerical simulations of

^E-mail: stuart.mcalpine@ helsinki.fi gas-rich major mergers have shown significant enhancements in star formation (e.g. Johansson, Burkert \& Naab 2009; Volonteri et al. 2015; Zolotov et al. 2015; Pontzen et al. 2017) and BH activity for at least one of the systems during the course of the interaction (e.g. Di Matteo, Springel \& Hernquist 2005; Springel, Di Matteo \& Hernquist 2005a). If the induced growth via the merging process were to contribute a significant fraction to the stellar and $\mathrm{BH}$ mass budgets, it could naturally give rise to the empirical scaling relations between the $\mathrm{BH}$ mass and various properties of the host galaxy, such as the velocity dispersion and mass of the stellar bulge (e.g. Magorrian et al. 1998; McConnell \& Ma 2013). Alternatively, the induced growth may be entirely non-consequential, with the correlations between $\mathrm{BHs}$ and their host galaxies only appearing as result of a random walk (e.g. Peng 2007; Hirschmann et al. 2010; Jahnke \& Macciò 2011). 
From an empirical point of view, the picture linking galaxy interactions to $\mathrm{BH}$ activity is less clear. At higher redshifts $(z$ $\gtrsim 1$ ), extremely luminous ( $L_{\mathrm{bol}} \geq 10^{46} \mathrm{erg} \mathrm{s}^{-1}$, where $L_{\mathrm{bol}}$ is the bolometric AGN luminosity) heavily obscured quasars are found to reside almost exclusively in disturbed systems, strongly in line with a merger-driven scenario (e.g. Glikman et al. 2015; Fan et al. 2016). However, Schawinski et al. (2012) see no such trend for similarly luminous AGN, finding the majority of their host galaxies to be disc-dominated, and therefore showing no sign of a recent interaction. Still at high redshift, low and intermediate luminosity AGN ( $L_{\mathrm{bol}} \leq 10^{45} \mathrm{erg} \mathrm{s}^{-1}$ ) typically exhibit merging fractions very similar to that of the inactive population (e.g. Schawinski et al. 2011; Kocevski et al. 2012; Rosario et al. 2015; Mechtley et al. 2016; Marian et al. 2019), suggesting that mergers have little influence towards enhancing $\mathrm{BH}$ activity in this regime.

The equivalent empirical studies at lower redshifts $(z \lesssim 1)$ are also mixed. Goulding et al. (2018) utilize a novel machine-learning technique applied to over 100000 spectroscopically confirmed systems in an attempt to automatically identify those with and without merging features. They find galaxies in the current state of a merger are $\approx 2-7$ times more likely to contain a luminous AGN than their non-interacting counterparts. This quantitatively agrees with previous studies, who also find a noticeable enhancement in the fraction of AGN that reside in either close pairs or morphologically disturbed hosts above a control sample (e.g. Koss et al. 2010; Ellison et al. 2011, 2013; Cotini et al. 2013; Satyapal et al. 2014; Ellison, Patton \& Hickox 2015; Rosario et al. 2015; Koss et al. 2018). Yet, again, many low-redshift studies also fail to find a distinction between the AGN fraction of interacting and noninteracting galaxies (e.g. Cisternas et al. 2011; Villforth et al. 2014; Hewlett et al. 2017; Villforth et al. 2017). Thus with the potential exception of extremely luminous AGN at high-redshift, it still remains unclear from observations what role galaxy-galaxy mergers have to play in triggering $\mathrm{BH}$ activity.

The discrepancies in the results between observational studies have been attributed to multiple factors. When trying to investigate correlations over a wide dynamic range of AGN luminosities, the small samples sizes of many of these studies can be particularly restrictive. More fundamentally, dust obscured AGN in merging systems may be missed entirely in surveys that only focus on shorter wavelengths (e.g. Goulding \& Alexander 2009; Weston et al. 2017; Koss et al. 2018), indicating that surveys in the infrared and rest frame hard x-rays may be the most effective measure of AGN selection (e.g. Brandt \& Alexander 2015). Perhaps most crucially, the process of identifying merging systems through morphological disturbances or asymmetry is especially challenging, and often done by eye (however this process is becoming increasingly automated with improving success, e.g. Pawlik et al. 2016; Goulding et al. 2018; Bottrell et al. 2019). As the surface brightness of tidal features is intrinsically low, particularly at high redshift and for low mass ratio interactions, many interacting systems may simply be misidentified as non-interacting. Similarly, resolving the final stages of the merger (the coalescence of the two galaxies nuclei), or identifying the signatures of galaxies immediately post-merger, are also extremely challenging, and require sensitive imaging. Finally, when selecting on a variable processes, such as AGN activity, any correlations that exist on average may be washed out entirely (Hickox et al. 2014), suggesting that a selection on both AGN activity and the merging indicators may be required for a fuller understanding (such as was done for Ellison et al. 2019, finding indeed that both mergers have an excess of AGN and AGN hosts are more frequently disturbed).
Hydrodynamical simulations of merging systems have provided compelling theoretical evidence for a link between BH activity and galaxy interactions (e.g. Dubois et al. 2015; Pontzen et al. 2017), yet the global significance of the merging process for boosting $\mathrm{BH}$ activity within a full cosmological context remains largely unknown. Steinborn et al. (2018) investigated the role of galaxy mergers as driving mechanisms for $\mathrm{BH}$ activity in the high mass regime $\left(M_{*} \geq 10^{11} \mathrm{M}_{\odot}\right)$ using the cosmological hydrodynamical MAGNETICUM PATHFINDER simulations. They argue, that whilst the merger fractions of AGN hosts can be up to three times higher than those of inactive galaxies, the role of mergers in high-mass galaxies are not statistically relevant for $\mathrm{BH}$ fuelling.

For this study we utilize EAGLE, a cosmological hydrodynamical simulation with more than an order of magnitude higher mass resolution than MAGNETICUM PATHFINDER, which has proven to reproduce many properties of the observed Universe with high fidelity: such as the colour bimodality of galaxies (Trayford et al. 2015), the evolution of galaxy sizes and star formation rates (Furlong et al. 2015, 2017), and the correlation between the star formation rate and $\mathrm{BH}$ activity (McAlpine et al. 2017; Scholtz et al. 2018). Here we build upon these successes, and investigate the connection between galaxy-galaxy mergers and $\mathrm{BH}$ activity.

The paper is organized as follows. In Section 2 we briefly overview the EAGLE simulation, our sample selection, and our control pairing criteria. Section 3 contains our results: investigating the merger fraction of AGN in Section 3.1.1, the AGN fraction of merging systems in Section 3.1.2, and the AGN fraction of close pairs in Section 3.1.3. We discuss our results, including a comparison to current observational studies, in Section 4, and finally conclude in Section 5.

\section{THE EAgLE SIMULATION}

EAGLE ('Evolution and Assembly of GaLaxies and their Environment'; Crain et al. 2015; Schaye et al. 2015) $)^{1,2}$ is a suite of cosmological smoothed particle hydrodynamics (SPH) simulations that cover a range of periodic volumes, numerical resolutions, and physical models. To incorporate the processes that operate below the simulation resolution a series of 'subgrid' prescriptions are implemented, namely: radiative cooling and photoionization heating (Wiersma, Schaye \& Smith 2009a); star formation (Schaye \& Dalla Vecchia 2008), stellar mass loss (Wiersma et al. 2009b), and stellar feedback (Dalla Vecchia \& Schaye 2012); BH growth via accretion and mergers, and BH feedback (Springel, Di Matteo \& Hernquist 2005b; Schaye et al. 2015; Rosas-Guevara et al. 2016). The free parameters of these models are calibrated to reproduce the observed galaxy stellar mass function, galaxy sizes, and the BH mass-bulge mass relation at $z \approx 0.1$. A full description of the simulation and the calibration strategy can be found in Schaye et al. (2015) and Crain et al. (2015), respectively.

For this study, we are interested in the influence of galaxy-galaxy mergers as triggering mechanisms for $\mathrm{BH}$ activity. Therefore to cover the widest dynamic range of AGN luminosities, Eddington rates, and host galaxy diversities, we restrict our study to the largest simulation, denoted Ref-L0100N1504. This simulation is a cubic

\footnotetext{
${ }^{1}$ www.eaglesim.org

${ }^{2}$ The galaxy and halo catalogues of the simulation suite, as well as the particle data, are publicly available at http://www.eaglesim.org/database.php (McAlpine et al. 2016; The EAGLE team 2017).
} 
periodic volume 100 comoving megaparsecs $(\mathrm{cMpc})$ on each side, sampled by $1504^{3}$ dark matter particles of mass $9.7 \times 10^{6} \mathrm{M}_{\odot}$ and an equal number of baryonic particles with an initial mass of $1.8 \times 10^{6} \mathrm{M}_{\odot}$. The subgrid parameters are those of the EAGLE reference model, described fully by Schaye et al. (2015). The simulation adopts a flat $\Lambda \mathrm{CDM}$ cosmogony with parameters inferred from the analysis of Planck data (Planck Collaboration XVI 2014): $\Omega_{\Lambda}=0.693, \Omega_{\mathrm{m}}=0.307, \Omega_{\mathrm{b}}=0.048, \sigma_{8}=0.8288, n_{\mathrm{s}}=0.9611$, and $H_{0}=67.77 \mathrm{~km} \mathrm{~s}^{-1} \mathrm{Mpc}^{-1}$. A Chabrier (2003) stellar initial mass function (IMF) is adopted.

The complete state of the simulation is stored at 400 intervals between redshift $z=20$ and $z=0$ in a series of data-lite 'snipshots'. In post-processing, the dark matter structure finding algorithm 'Friends of Friends' and the substructure finding algorithm SUBFIND (Springel et al. 2001; Dolag et al. 2009) were performed on 200 of these outputs to produce a set of halo and galaxy catalogues. The galaxies are then tracked through cosmic time via a merger tree, with the history of each galaxy being considered from the reference frame of their main progenitor, defined as the branch of the galaxy's full merger tree that contains the greatest total mass (see Qu et al. 2017, for full details).

Halo mass, $M_{200}$, is defined as the total mass enclosed within $r_{200}$, the radius at which the mean enclosed density is 200 times the critical density of the Universe (i.e. $200 \rho_{\text {crit }}$ ). Galaxy mass, $M_{*}$, is defined as the total stellar content bound to a subhalo within a spherical aperture with radius 30 proper kiloparsecs (pkpc), as per Schaye et al. (2015).

\subsection{The BH subgrid model}

The most influential subgrid models for this study are those that govern the behaviour of $\mathrm{BHs}$, and therefore here we briefly outline their implementation. For a complete description of these models see Schaye et al. (2015) and Rosas-Guevara et al. (2015), to see how BHs were considered during the calibration strategy see Crain et al. (2015).

BHs are initially seeded with a mass of $m_{\text {seed }}=1.48 \times 10^{5} \mathrm{M}_{\odot}$ into dark matter haloes of mass $M_{\text {halo }}=1.48 \times 10^{10} \mathrm{M}_{\odot}$ that do not already contain a $\mathrm{BH}$. The $\mathrm{BHs}$ are then free to grow via the Eddington limited accretion of neighbouring gas using a modified Bondi-Hoyle (Bondi \& Hoyle 1944) formalism that accounts for the angular momentum of the gas (Rosas-Guevara et al. 2015), i.e.

$\dot{m}_{\mathrm{BH}}=\dot{m}_{\text {bondi }} \times \min \left(C_{\mathrm{visc}}^{-1}\left(c_{\mathrm{s}} / V_{\phi}\right)^{3}, 1\right)$,

where $\dot{m}_{\text {bondi }}$ is the Bondi \& Hoyle (1944) rate for spherically symmetric accretion,

$\dot{m}_{\text {bondi }}=\frac{4 \pi G^{2} m_{\mathrm{BH}}^{2} \rho}{\left(c_{s}^{2}+v^{2}\right)^{3 / 2}}$.

Here, $m_{\mathrm{BH}}$ is the mass of the $\mathrm{BH}, \rho$ is the density of the surrounding gas, $c_{s}$ is the sound speed of the surrounding gas, $v$ is the relative velocity of the $\mathrm{BH}$, and the surrounding gas and $V_{\phi}$ is the rotation speed of the surrounding gas. $C_{\text {visc }}$ is a free parameter related to the viscosity of the (subgrid) accretion disc (see Rosas-Guevara et al. 2015). BHs also grow via mergers with neighbouring BHs. This occurs instantaneously when two BHs overlap to within each others smoothing kernel (equating to a median separation of $\approx 1 \mathrm{pkpc}$ at all redshifts) and their relative velocity to one another is less than the circular velocity at that distance (see Salcido et al. 2016, for a detailed description of this process). The feedback from BHs is implemented using only a single mode, whereby energy is injected thermally and stochastically into the surrounding gas, raising their temperature by a fixed increment.

We note that during the calibration of the subgrid models the observed BH mass-stellar mass relation at $z \approx 0$ was deliberately achieved (Crain et al. 2015). However, the influence of mergers upon $\mathrm{BH}$ growth was never considered during this process, and thus is a direct prediction of the simulation. The EAGLE simulation under this setup has produced an overall realistic $\mathrm{BH}$ population (e.g. Schaye et al. 2015; Rosas-Guevara et al. 2016), capable of matching many observed relations and behaviours (e.g. McAlpine et al. 2016; Rosas-Guevara et al. 2016; Scholtz et al. 2018).

\subsection{Galaxy-galaxy mergers}

A galaxy is said to have undergone a merger within the simulation if two independent bound dark matter haloes from a simulation output go on to become a single bound dark matter halo in the next simulation output (bound as defined by the SUBFIND algorithm; see Qu et al. 2017, for more details). We therefore know the cosmic time of coalescence between two galaxies, denoted $t_{\text {merger }}$, to within the temporal spacing of the simulation outputs (i.e. to within $\approx 50 \mathrm{Myr}$ ), and we assign a random cosmic time between the two outputs for the value of $t_{\text {merger }}$. The mergers between two galaxies are classified by the stellar mass ratio, $M_{*, 1} / M_{*, 2}$, where $M_{*, 2}$ is always set to be the stellar mass of the most massive member of the galaxy pair. A merger is considered to be 'major' if $M_{*, 1} / M_{*, 2} \geq \frac{1}{4}$ and 'minor' if $\frac{1}{10}<M_{*, 1} / M_{*, 2}<\frac{1}{4}$. To account for the stellar stripping that occurs during the later stages of the interaction, the stellar mass ratio is computed when the galaxy in-falling on to the main progenitor had its maximum mass (e.g. Rodriguez-Gomez et al. 2015; Qu et al. 2017).

Following McAlpine et al. (2018), we parametrize the "merging state' of a galaxy by its value of $n_{\text {dyn }}$, defined as the number of dynamical times to the nearest, i.e. the most proximate in time, merger, i.e.

$n_{\mathrm{dyn}} \equiv \frac{t-t_{\mathrm{merger}[\text { nearest }]}}{t_{\mathrm{dyn}}}$,

where $t$ is the cosmic time at which the galaxy was sampled (i.e. the cosmic time of the simulation output), $t_{\text {merger[nearest] }}$ is the cosmic time of the most proximate in time merger, and $t_{\mathrm{dyn}}$ is the dynamical time at the time $t$, defined as the free-fall time of a dark matter halo, i.e.

$t_{\text {dyn }} \equiv\left(\frac{3 \pi}{32 G\left(200 \rho_{\text {crit }}\right)}\right)^{1 / 2}$.

For reference, the dynamical time is $\approx 1.6 \mathrm{Gyr}$ at $z=0, \approx 0.5 \mathrm{Gyr}$ at $z=2$ and $\approx 0.2 \mathrm{Gyr}$ at $z=5$. A positive value of $n_{\text {dyn }}$ indicates that the galaxy's most proximate in time merger will complete $n$ dynamical times in the future, whilst a negative value of $n_{\text {dyn }}$ indicates that the galaxy's most proximate in time merger has already completed, and was $n$ dynamical times in the past. If a galaxy has a value $\left|n_{\text {dyn }}\right| \leq 1$ (i.e. it will complete or has completed a merger within one dynamical time) we define the galaxy to be 'in the state of a merger'. We compute $n_{\mathrm{dyn}}$ separately for the most proximate in time major merger and the most proximate in time minor merger, denoted $n_{\text {dyn[Major] }}$ and $n_{\text {dyn[Minor] }}$, respectively. We chose to operate in a fixed window of dynamical time to define our merging state, over a fixed window of cosmic time, to more fairly compare results from a range of redshifts whilst incorporating the evolving dynamical state of the Universe. We acknowledge that the duration of one dynamical time at low redshift is longer than the 
Table 1. An overview of the four samples used throughout this study, showing their selection criteria, the unique selection criteria of their associated control sample, the mean number of galaxies that meet each selection criteria per simulation output and the results section the sample is used for. For the 'Major mergers' sample, $\left|n_{\mathrm{dyn}[\mathrm{Major}]}\right| \leq 1$ refers to galaxies that have completed or will complete a major merger within \pm 1 dynamical time, i.e. they are 'in the state of a major merger' (see Section 2). For the 'Close pairs' sample, $r_{\text {sep[Major] }}$ refers to the $3 \mathrm{D}$ distance to the closest major companion. 'Major' in all cases refers to a stellar mass ratio of $M_{*, 1} / M_{*, 2} \geq \frac{1}{4}$, where $M_{*, 2}$ is always set to be the stellar mass of the most massive member of the galaxy pair. Note only galaxies with stellar masses $M_{*} \geq 10^{10} \mathrm{M}_{\odot}$ in the redshift range $0<z<5$ are considered for each sample.

\begin{tabular}{|c|c|c|c|c|c|c|}
\hline \multirow[t]{2}{*}{ Sample } & \multirow{2}{*}{ Selection criteria } & \multirow{2}{*}{ Unique control criteria } & \multicolumn{3}{|c|}{$\langle N\rangle$} & \multirow{2}{*}{ Reference } \\
\hline & & & $0<z<1$ & $1<z<2$ & $2<z<5$ & \\
\hline AGN luminosity & $L_{\mathrm{bol}} \geq 10^{43} \mathrm{erg} \mathrm{s}^{-1}$ & $L_{\mathrm{bol}}<10^{43} \mathrm{erg} \mathrm{s}^{-1}$ & 373 & 589 & 269 & Section 3.1.1 \\
\hline Eddington rate & $\lambda_{\text {edd }} \geq 10^{-2}$ & $\lambda_{\text {edd }}<10^{-2}$ & 263 & 472 & 206 & Section 3.1.1 \\
\hline Major mergers & $\left|n_{\text {dyn }[\text { Major }]}\right| \leq 1$ & $\left|n_{\text {dyn[Major] }}\right|>2$ & 410 & 467 & 166 & Section 3.1 .2 \\
\hline Close pairs & $r_{\text {sep }[\text { Major }]} \leq 100 \mathrm{pkpc}$ & $r_{\text {sep }[\text { Major }]}>200 \mathrm{pkpc}$ & 424 & 443 & 130 & Section 3.1.3 \\
\hline
\end{tabular}

time-scale that is commonly considered for the direct influence of mergers upon AGN activity $(\approx 0.5 \mathrm{Gyr}$; e.g. Hopkins et al. 2008; Johansson et al. 2009; Steinborn et al. 2018). However, in this study we do find evidence of AGN enhancement at lower redshifts up to one dynamical time after the merger has completed (see Section 3.3). Regardless, it should be noted that the choice of dynamical time window has a very limited impact on our overall results (see Appendix A).

\subsection{Sample selection}

Four mock galaxy samples are constructed for the analysis in Section 3 (also summarized in Table 1):

(i) $L_{\text {bol }}$ selected: all galaxies hosting a $\mathrm{BH}$ with a bolometric AGN luminosity ${ }^{3}$ greater than $L_{\mathrm{bol}} \geq 10^{43} \mathrm{erg} \mathrm{s}^{-1}$.

(ii) Eddington rate selected: all galaxies hosting a $\mathrm{BH}$ with an Eddington rate greater than $^{4}$ edd $\geq 10^{-2}$.

(iii) Major mergers: all galaxies currently in the state of a major merger, i.e. those with a value $\left|n_{\text {dyn[Major }]}\right| \leq 1$, where major refers to a stellar mass ratio of $M_{*, 1} / M_{*, 2} \geq \frac{1}{4}$.

(iv) Close pairs: all galaxies with a major companion. i.e. those with a companion with a stellar mass ratio of $M_{*, 1} / M_{*, 2} \geq \frac{1}{4}$, within a 3D physical separation of $r_{\mathrm{sep} \text { [Major] }} \leq 100$ proper kiloparsecs (pkpc).

Each sample is designed to investigate how mergers influence $\mathrm{BH}$ activity from complementary perspectives, analysed separately in Sections 3.1.1 to 3.1.3. We limit our selections to the redshift range $0<z<5$. To ensure that minor mergers remain well resolved (for the discussion in Section 4.3), for each sample we only consider galaxies with stellar masses greater than $M_{*} \geq 10^{10} \mathrm{M}_{\odot}$ (i.e. $M_{*, 1}$ $\geq 10^{9} \mathrm{M}_{\odot}$ ). The final samples are constructed by combining the galaxies from each simulation output that lie within the desired redshift range.

\subsubsection{Constructing a control sample}

In order to establish the influence of galaxy mergers upon enhanced $\mathrm{BH}$ activity, for each of the four samples outlined above we require

\footnotetext{
${ }^{3}$ The bolometric AGN luminosity if defined as $L_{\mathrm{bol}}=\epsilon_{r} \dot{m}_{\mathrm{BH}} c^{2}$, where $c$ is the speed of light, $\dot{m}_{\mathrm{BH}}$ is the accretion rate of the $\mathrm{BH}$ and $\epsilon_{r}$ is the radiative efficiency of the accretion disc, which is assumed to be 0.1 (Shakura \& Sunyaev 1973).

${ }^{4}$ The Eddington rate is defined as $\lambda_{\text {edd }}=L_{\text {bol }} / L_{\text {edd }}$, where $L_{\text {edd }}$ is the Eddington luminosity.
}

a suitably constructed control. Therefore for each selected galaxy, we assign to it a single randomly selected control galaxy. How one selects the control galaxies is not necessarily straightforward, and must reflect the science question that is being asked. For example, when investigating the influence of mergers for creating active galaxies (i.e. $L_{\mathrm{bol}} \geq 10^{43} \mathrm{erg} \mathrm{s}^{-1}$ or $\lambda_{\text {edd }} \geq 10^{-2}$ ), we wish to contrast the behaviours against a control set of inactive galaxies (i.e. $L_{\mathrm{bol}}$ $<10^{43} \mathrm{erg} \mathrm{s}^{-1}$ or $\lambda_{\text {edd }}<10^{-2}$ ). In addition, we must ensure that the control galaxies are as similar as possible in their integrated properties to the selected galaxies in order to provide the fairest comparison. Typically, the control galaxies for studies of this nature are only paired on their stellar mass and redshift, to account for the known evolution of the merger fraction with both redshift and mass (e.g. Rodriguez-Gomez et al. 2015; Qu et al. 2017). However, there are many other properties of galaxies that could also influence the growth behaviour of BHs: for example the available gas content, BH mass, or environmental properties: such as the halo mass $\left(M_{200}\right)$, or the $N_{2}$ and $r_{2}$ parameters (defined for this study as the number of major, $M_{*, 1} / M_{*, 2} \geq \frac{1}{4}$, companions within $2 \mathrm{pMpc}$, and the distance to the 2 nd closest major companion, respectively, similar to the methods of observational studies; e.g. Ellison et al. 2010; Patton et al. 2013, 2016). We note that we would always argue against matching on the SFR, as the SFR of a galaxy can also be enhanced during the merger process (e.g. Rodríguez Montero et al. 2019).

To ensure that the control galaxies are as similar as possible to the selected galaxies, we opt for the following matching criteria: the control galaxy must be taken from the same simulation output (i.e. it has the same redshift, $z)$, have a stellar mass $\left(M_{*}\right)$, halo mass $\left(M_{200}\right)$, gas mass $\left(M_{\mathrm{gas}}\right), \mathrm{BH}$ mass $\left(M_{\mathrm{BH}}\right)$, and value of $r_{2}$ to within $0.05 \mathrm{dex}$ of the selected galaxy, and have a value of $N_{2}$ to within 5 percent of the selected galaxy. On top of this, each sample has an additional unique control criteria condition depending on the science question that is being asked (listed in Table 1). If multiple galaxies meet these criteria, one galaxy is selected at random to be the control. However if no suitable control galaxy is found, the matching criteria is progressively loosened by increments of 0.05 dex ( 5 per cent for $N_{2}$ ), up to a maximum of $0.3 \mathrm{dex}$ ( 30 per cent for $N_{2}$ ), until a suitable control galaxy is found. If there still remains no suitable control galaxy after this process, then the galaxy is discarded from the sample (resulting in 3-7 percent of the sample being discarded depending on redshift). We note that for the close pairs sample we match $r_{1}$ of the control galaxy to $r_{2}$ of the selected galaxy (i.e. the distance to the second closest major companion of the selected galaxy must match the distance to the closest major companion of the control galaxy, as per Ellison et al. 2010; Patton et al. 2013, 2016). 
We acknowledge that these matching criteria are beyond current observational capabilities, but employ them for the analysis in Section 3 to see what role mergers play in triggering AGN activity using the strictest control sets. We investigate how the choice of matching criteria affects the results in Appendix A2, and discuss what impact this may have when trying to recover any trends observationally in Section 4.2.

The control galaxies matched to the selected galaxies from the four samples are combined to construct four associated control samples, which are designed to trace the underlying merger rate (or AGN fraction) of similar galaxies, whilst remaining as independent as possible from the original sample selection. Any trends that deviate from the trends of the control samples tells us how mergers are influencing $\mathrm{BH}$ activity in the simulation.

\subsection{The merger fraction}

The merger fraction of AGN is defined as the number of AGN with a value of $n_{\text {dyn }}$ that lie within a chosen window, divided by the total number of AGN, i.e.

$f_{\text {merger,AGN }}=\frac{N_{\mathrm{AGN}}\left[a \leq n_{\mathrm{dyn}} \leq b\right]}{N_{\mathrm{AGN}}}$,

where $a$ and $b$ are the minimum and maximum values of $n_{\text {dyn }}$ that the AGN can have to still be considered in a merging state. Our fiducial values are $a=-1$ and $b=1$, i.e. an AGN is considered to be 'within the state of a merger' if it is within \pm 1 dynamical time from coalescence of the two galaxies. The merger fraction of the control sample $\left(f_{\text {merger, control }}\right)$ is defined in the same manner, now considering what fraction of the associated control galaxies have values of $n_{\text {dyn }}$ between $a$ and $b$ divided by the total number of control galaxies. The excess in the merger fraction is simply the ratio of these two fractions (excess $=f_{\text {merger, AGN }} / f_{\text {merger, control }}$ ). We note for the figures in Section 3 we convert $f_{\text {merger, AGN }}$ to a percentage for clarity.

We report errors on the merger fraction as the Poisson error, i.e. the numerator in equation (5) is replaced with the square root. As we quote the merger fraction as integer percentages, any error below 0.5 percent is reported as 0 percent.

\section{RESULTS}

\subsection{The enhancement in $\mathrm{BH}$ activity due to major mergers}

We begin with an investigation to see if there is a measurable excess in $\mathrm{BH}$ activity during the period of a major merger. We do this via three methods, exploring: the merger fraction of AGN in Section 3.1.1, the AGN fraction of merging systems in Section 3.1.2, and the AGN fraction of close pairs in Section 3.1.3. Each method tackles the question from a complementary, yet alternative approach, each using a unique galaxy sample and associated control sample, outlined in Table 1.

For the analysis below, the samples are split into galaxies at 'low' $(0<z<1)$, 'intermediate' $(1<z<2)$, and 'high' redshift $(2<z<5)$ to avoid misinterpreting any behavioural trend with the underlying evolution of the merger fraction through cosmic time (e.g. Rodriguez-Gomez et al. 2015; Qu et al. 2017). As a reminder, each sample only contains galaxies more massive than $M_{*} \geq 10^{10} \mathrm{M}_{\odot}$, and here we are only considering the influence of major mergers (i.e. those with stellar mass ratios of $M_{*, 1} / M_{*, 2} \geq \frac{1}{4}$ ).

We caution that the results from this section should not be directly compared to observational studies in a quantitative sense, as the resulting merger and AGN fractions quoted below are sensitive to our definitions of a 'merging state' and 'active BH' (see Appendix A). Furthermore, the galaxy properties chosen to match the selected galaxies to their control galaxies also has an impact on the results (see Appendix A2), and here were have selected a strict criterion beyond the capabilities of current observational studies. We can however compare the results of this section to observational studies in a qualitative sense, which we discuss in Section 4.2. In addition, in Section 4.2.1 we emulate the observed selection and control pairing criteria of the AGN fractions of close pairs and quantitatively compare the results from the simulation to the observational studies.

\subsubsection{The merger fraction of $A G N$}

The left-hand panel of Fig. 1 shows the AGN major merger fraction (i.e. the fraction of AGN hosted by galaxies in the state of a major merger) as a function of the bolometric AGN luminosity. Alongside, the merger fraction of the associated control sample of inactive galaxies is also shown. We note that the control galaxies are linked to the galaxies within the active sample using our matching criteria (see Section 2.3.1), and are presented on the figure using the luminosity or Eddington rate of their associated active galaxy (whilst themselves being inactive galaxies). The merger fraction of the control sample represents the predicted baseline for similar, yet inactive, galaxies, with any deviation from this baseline highlighting the influence of major mergers upon increased $\mathrm{BH}$ activity.

The merger fraction of both the AGN and the control galaxies systematically increase with increasing redshift, which is true also for the general population at a fixed mass $\left(\propto(1+z)^{2.4-2.8}\right.$; e.g. Rodriguez-Gomez et al. 2015). Within each redshift range, we find an increasing merger fraction with increasing AGN luminosity: rising from $\approx 17 \pm 0$ per cent $\rightarrow 33 \pm 5$ per cent in the redshift range $0<z<1, \approx 21 \pm 1$ per cent $\rightarrow 40 \pm 5$ per cent in the redshift range $1<z<2$, and $\approx 26 \pm 1$ percent $\rightarrow 41 \pm 3$ percent in the redshift range $2<z<5$ (each reported for the luminosity range $1 \times 10^{43} \leq$ $\left.L_{\mathrm{bol}} \leq 2 \times 10^{45} \mathrm{erg} \mathrm{s}^{-1}\right)$. The merger fraction of the matched sample of inactive control galaxies similarly increases alongside the AGN sample (due to them being matched on mass, see below). However, due to the shallower gradient in the trends of the control samples, an increasing offset between the two populations emerges. This excess is quantified in the lower panel, showing the ratio of the merger fraction between the active and inactive populations. As the AGN luminosity increases, so too does the excess in the merger fraction, reaching a value of $\approx 1.75$ for the highest luminosities we can explore. This suggests that the brightest AGN, particularly those above $L_{\text {bol }} \sim 10^{45} \mathrm{erg} \mathrm{s}^{-1}$, reside more often within merging systems over their isolated counterparts. It is also worth noting that even lower luminosity AGN $\left(L_{\text {bol }} \sim 10^{43} \mathrm{erg} \mathrm{s}^{-1}\right)$ at lower redshifts $(0<$ $z<1$ ) exhibit an excess in their merger fraction, which agrees with observations of lower luminosity Seyferts in the local Universe (e.g. Ellison et al. 2011, 2013, 2015).

The increasing merger fraction with increasing AGN luminosity seen in the left-hand panel of Fig. 1 is, in part, also driven by mass. This is because whenever we consider the most luminous AGN, we are typically biased towards more massive BHs (which typically reside in more massive galaxies, and the merger fraction of galaxies increases with increasing mass at a fixed redshift; e.g. RodriguezGomez et al. 2015; Qu et al. 2017). Indeed, the BHs in the redshift range $0<z<1$ that occupy the lowest AGN luminosity bin in Fig. 1 have a median mass of $M_{\mathrm{BH}} \approx 3 \times 10^{7} \mathrm{M}_{\odot}$ (hosted by galaxies with 

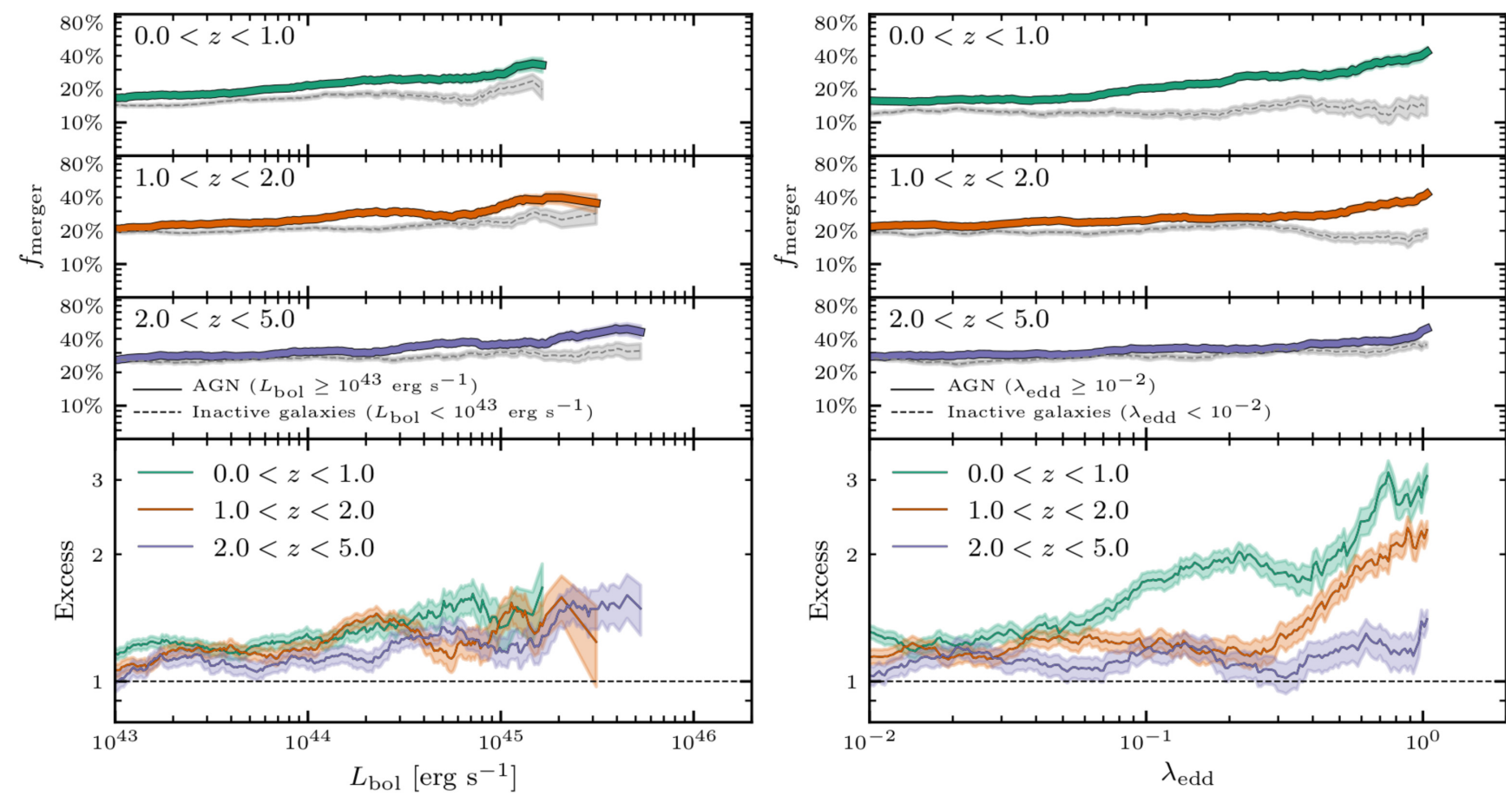

Figure 1. The AGN major merger $\left(M_{*, 1} / M_{*, 2} \geq \frac{1}{4}\right)$ fraction as a function of the bolometric AGN luminosity (left-hand panel) and the Eddington rate (right-hand panel). In both panels, the major merger fraction of the associated matched sample of inactive galaxies acts as our control (see Section 2.3.1). We note that the control galaxies are linked to the galaxies within the active sample using our matching criteria (see Section 2.3.1), and are presented on the figure using the luminosity or Eddington rate of their associated active galaxy (whilst themselves being inactive galaxies). The shaded regions represent the Poisson uncertainty. For both metrics of BH activity, and for each redshift range, the merger fraction of AGN increases with increasing AGN luminosity or Eddington rate, and is typically higher than the merger fractions of the inactive control samples. The excess between the merger fraction of the AGN and the inactive galaxies is shown in the lower panels: reaching a maximum value of $\approx 1.75$ at high AGN luminosities, and reaching a maximum value of $\approx 3$ at high Eddington rates. This indicates that there is more high-luminosity/Eddington rate AGN in a merging state relative to similar inactive galaxies. This enhancement persists out to the highest redshifts we explore, but is typically more prominent at low redshift (particularly in the case of the Eddington rate). The increased excess when considering the Eddington rate, rather than the AGN luminosity, suggests that it is a clearer indicator of the enhancement of BH activity during mergers.

a median mass of $M_{*} \approx 3 \times 10^{10} \mathrm{M}_{\odot}$ ), whereas the BHs in the highest luminosity bin have a median mass of $M_{\mathrm{BH}} \approx 2 \times 10^{8} \mathrm{M}_{\odot}$ (hosted by galaxies with a median mass of $M_{*} \approx 1 \times 10^{11} \mathrm{M}_{\odot}$ ). This mass differential from the low- to high-luminosity end is why the merger fraction of the (stellar and BH mass matched) control galaxies also increases.

If we consider the mass bias inherent to the AGN luminosities, it is therefore potentially more informative to investigate the major merger fraction of AGN as a function of a $\mathrm{BH}$ mass weighted property, such as the Eddington rate. Using this metric, we can more fairly identify the BHs with atypically high (or low) accretion rates, independent of their mass. We investigate the AGN major merger fraction as a function of the Eddington rate in the right-hand panel of Fig. 1, finding similar overall trends to the bolometric AGN luminosity in the left-hand panel: rising from $\approx 16 \pm 0$ percent $\rightarrow 43 \pm 4$ percent in the redshift range 0 $<z<1, \approx 22 \pm 1$ percent $\rightarrow 43 \pm 3$ percent in the redshift range $1<z<2$, and $\approx 28 \pm 1$ percent $\rightarrow 50 \pm 2$ percent in the redshift range $2<z<5$ (each reported for the Eddington rate range $10^{-2} \leq \lambda_{\text {edd }} \leq 1.48^{5}$ ). The excess in the merger fraction relative to the inactive control galaxies are also similar to the trends found for the bolometric luminosity in the left-hand panel, with the

${ }^{5}$ The $\mathrm{BH}$ accretion rate in the EAGLE reference model is capped to the Eddington limit over $h$ (i.e. the maximum allowed value of $\lambda_{\text {edd }}=1 / h=$ 1.48). exception of high Eddington rate galaxies at lower redshifts $(z<$ 2 ), where the excess now reaches values of $\approx 2-3$. This increased excess at lower redshifts is in part due to the slight downturn of the merger fraction of the control galaxies beyond $\lambda_{\text {edd }} \approx 3 \times 10^{-1}$, created from the increasing dominance of lower mass galaxies over their more massive counterparts with increasing Eddington rate.

We note that the merger fractions in the upper panels of Fig. 1, and the resulting value of the fractional excess shown in the lower panel of Fig. 1, are sensitive to our definition of a 'merging state'. In Appendix A we explore how the choice of dynamical time window used to define the state of a merger (which for this study was chosen to be \pm 1 dynamical time, i.e. $a=-1$ and $b=1$ from equation 5) influences the resulting excess values, finding that shorter dynamical time windows typically result in larger excess values (by up to a factor of $\approx 2$, but is often much less, see Fig. A1). However, the overall behaviour in the trends (i.e. an increasing excess with increasing AGN luminosity or Eddington rate) is not impacted by the choice of dynamical time window.

Thus we find that AGN are more commonly found in merging systems over their inactive counterparts. The excess signal is most prominent in two cases: (1) from luminous $\left(L_{\mathrm{bol}} \geq 10^{45} \mathrm{erg} \mathrm{s}^{-1}\right.$ ) massive BHs $\left(M_{\mathrm{BH}} \sim 10^{8} \mathrm{M}_{\odot}\right)$, where the excess in the merger fraction reaches a factor of $\approx 1.75$, and (2) from less massive $\mathrm{BHs}$ $\left(M_{\mathrm{BH}} \sim 10^{6} \mathrm{M}_{\odot}\right)$ accreting close to the Eddington limit, where the excess in the merger fraction reaches a value of $\approx 1.5-3$. Selecting $\mathrm{BHs}$ by the Eddington rate appears to provide a fairer view of 
how mergers influence $\mathrm{BH}$ activity, as it can more fairly include the contribution from lower mass BHs/galaxies whose intrinsically low AGN luminosities (but high Eddington rates) are typically lost to the background of regularly accreting more massive BHs/ galaxies.

\subsubsection{The AGN fraction of merging systems}

When forming correlations between a stochastic process, such as $\mathrm{BH}$ accretion, and a typically stable process, such as the evolution of galaxy wide properties, it has been argued that by initially selecting on the highly variable process one could inadvertently wash out or dilute any underlying correlations that exist between the two processes on average (e.g. Hickox et al. 2014). For this reason, here we investigate the reverse of our approach in Section 3.1.1, that is, rather than considering the merger fraction of AGN, we now consider the AGN fraction of merging systems. Here, a $\mathrm{BH}$ is considered 'active' if it has a bolometric luminosity above $L_{\mathrm{bol}}$ $\geq 10^{43} \mathrm{erg} \mathrm{s}^{-1}$ or an Eddington rate above $\lambda_{\text {edd }}>10^{-2}$, although we test how the choice of higher limits affects the results in Appendix A. To establish the importance of any discovered trend, we again require a control sample. Therefore for each merging system, we match it with a similar isolated control galaxy (see Section 2.3.1), in order to quantify, at fixed $M_{*}$ and $z$, how the AGN fractions of merging and isolated galaxies compare.

The left-hand panel of Fig. 2 shows, as a function of the stellar mass, the fraction of major mergers (i.e. $\left|n_{\text {dyn[Major] }}\right| \leq 1$ ) and isolated systems (i.e. $\left|n_{\text {dyn[Major] }}\right|>2$ ) that host a $\mathrm{BH}$ with a bolometric AGN luminosity $L_{\text {bol }} \geq 10^{43} \mathrm{erg} \mathrm{s}^{-1}$. At fixed stellar mass, the AGN fraction of both merging and isolated systems systematically decreases with decreasing redshift (commonly referred to as AGN 'downsizing'; e.g. Hirschmann et al. 2014). Within each redshift range, the AGN fraction of merging galaxies increases with increasing stellar mass: rising from $\approx 8 \pm 1$ per cent $\rightarrow 41 \pm 5$ per cent in the redshift range $0<z<1, \approx 17 \pm 1$ per cent $\rightarrow 62 \pm 12$ per cent in the redshift range $1<z<2$, and $\approx 41 \pm 1$ per cent $\rightarrow 73 \pm 16$ per cent in the redshift range $2<z<5$ (each reported for the stellar mass range $1 \times 10^{10} \leq M_{*} \leq 1 \times 10^{11} \mathrm{M}_{\odot}$ ). These upward trends simply reflect the fact that more massive galaxies typically host more massive $\mathrm{BHs}$, and as the mass of the $\mathrm{BH}$ increases, a luminosity greater than $10^{43} \mathrm{erg} \mathrm{s}^{-1}$ can more easily be achieved. It is for the same reason that an upward trend in the AGN fraction is also emulated by the isolated control galaxies.

The excess between the AGN fraction of merging and isolated systems is shown in the lower panel of Fig. 2. For each redshift range, the excess increases with decreasing stellar mass, up to a maximum value of $\approx 1.8$ for galaxies with stellar masses $M_{*} \approx$ $10^{10} \mathrm{M}_{\odot}$ at $z=0$. At higher stellar masses $\left(M_{*} \gtrsim 10^{11} \mathrm{M}_{\odot}\right)$, there is little evidence for any excess in the AGN fraction. The potential lack of excess in more massive systems could be caused by our choice of AGN limit, as the most massive BHs residing in the most massive galaxies may simply naturally accrete above $L_{\mathrm{bol}}$ $\geq 10^{43} \mathrm{erg} \mathrm{s}^{-1}$ regardless of the merging state (erasing any excess). This does not appear to be the case, however, as even when the AGN limit is increased, the excess remains primarily in galaxies below $M_{*} \lesssim 10^{11} \mathrm{M}_{\odot}$ (see Fig. A2).

If we now consider the fraction of merging and isolated galaxies that host BHs with high Eddington rates $\left(\lambda_{\text {edd }} \geq 10^{-2}\right.$, shown in the right-hand panel of Fig. 2), we find a decreasing trend with increasing stellar mass: declining from $\approx 17 \pm 1$ per cent $\rightarrow 5 \pm 0$ per cent in the redshift range $0<z<1, \approx 29 \pm 1$ per cent $\rightarrow 16 \pm 1$ per cent in the redshift range $1<z<2$ and $\approx 47 \pm 2$ percent $\rightarrow$ $34 \pm 6$ percent in the redshift range $2<z<5$ (each reported for the stellar mass range $1 \times 10^{10} \leq M_{*} \leq 1 \times 10^{11} \mathrm{M}_{\odot}$ ). This trend is formed, again, from that of an increasing BH mass with increasing stellar mass, and whilst high luminosities are common for massive BHs, high Eddington rates become increasingly rare. As with the AGN luminosities, the excess between the merging and isolated systems increases with decreasing stellar mass at lower redshifts $(z<1)$, but is approximately constant at all stellar masses at higher redshifts $(z>1)$. Unlike in Fig. 1, where the Eddington rate revealed a larger signal in the excess relative to the AGN luminosity, here both the AGN fraction classified by the AGN luminosity or Eddington rate yield similar values.

Thus we see further evidence that major mergers trigger an increased amount of $\mathrm{BH}$ activity, and, as with Fig. 1, the excess above the control sample appears to be greatest at lower redshifts $(z<1)$. We note that the choice of AGN luminosity or Eddington rate cut used to classify an AGN (which was $L_{\text {bol }} \geq 10^{43} \mathrm{erg} \mathrm{s}^{-1}$ or $\lambda_{\text {edd }}>10^{-2}$ in Fig. 2) does directly impact the excess values, with higher cuts resulting in a greater excess above the control sample of isolated galaxies (see Fig. A2). This suggests that the most luminous and highest Eddington rate AGNs are more strongly linked with interactions (which was also seen in Fig. 1).

\subsubsection{The AGN fraction of close pairs}

Our final method of analysis investigates the AGN fraction of galaxies with a close major companion (i.e. a companion with a stellar mass ratio of $M_{*, 1} / M_{*, 2} \geq \frac{1}{4}$ ) within a $3 \mathrm{D}$ distance of $r_{\text {sep[Major] }} \leq 100 \mathrm{pkpc}$ (note that pkpc still refers to proper kiloparsecs and not projected kiloparsecs). For a control, we match each galaxy that has a close major companion to a similar 'isolated' galaxy (i.e. one that does not have a major companion within $200 \mathrm{pkpc}$, see Section 2.3.1). We note that the isolated control galaxies are linked to the galaxies within the close pairs sample using our matching criteria (see Section 2.3.1), and are presented on the figures using the 3D separation of the close pair galaxy (whilst themselves having no close major companions within $200 \mathrm{pkpc}$ ). As with the previous section, a galaxy is defined to host an AGN if the BH has a bolometric luminosity in excess of $L_{\mathrm{bol}} \geq 10^{43} \mathrm{erg} \mathrm{s}^{-1}$ or an Eddington rate in excess of $\lambda_{\text {edd }} \geq 10^{-2}$ (however we test the effect of different cuts in Appendix A).

The left-hand panel of Fig. 3 investigates the AGN fraction of galaxies with a close major companion as a function of the $3 \mathrm{D}$ pair separation, where an AGN is defined by a cut in the bolometric luminosity (i.e. $L_{\mathrm{bol}} \geq 10^{43} \mathrm{erg} \mathrm{s}^{-1}$ ). There is a weak trend of an increasing AGN fraction with decreasing pair separation: rising from $\approx 10 \pm 0$ per cent $\rightarrow 16 \pm 1$ per cent in the redshift range $0<$ $z<1, \approx 24 \pm 1$ percent $\rightarrow 32 \pm 1$ percent in the redshift range 1 $<z<2$, and $\approx 40 \pm 2$ per cent $\rightarrow 44 \pm 2$ per cent in the redshift range $2<z<5$ (each reported for the 3D separation range 100 $\geq r_{\text {sep[Major] }} \geq 10 \mathrm{pkpc}$ ). The galaxies within the control samples exhibit a very similar upward trend with decreasing pair separation, resulting in only a marginal excess between the AGN fraction of the close pair galaxies and the isolated control galaxies (hovering around excess values of $\approx 1.1$ for separations $r_{\text {sep[Major] }} \lesssim 80 \mathrm{pkpc}$ at $z<1$, shown in the lower panel). The scenario of the galaxies with the closest companions having the highest AGN fractions would presumably point towards further evidence of a triggering influence of interactions upon enhanced $\mathrm{BH}$ activity. However, in this case the dominant reason for an increasing AGN fraction with decreasing 

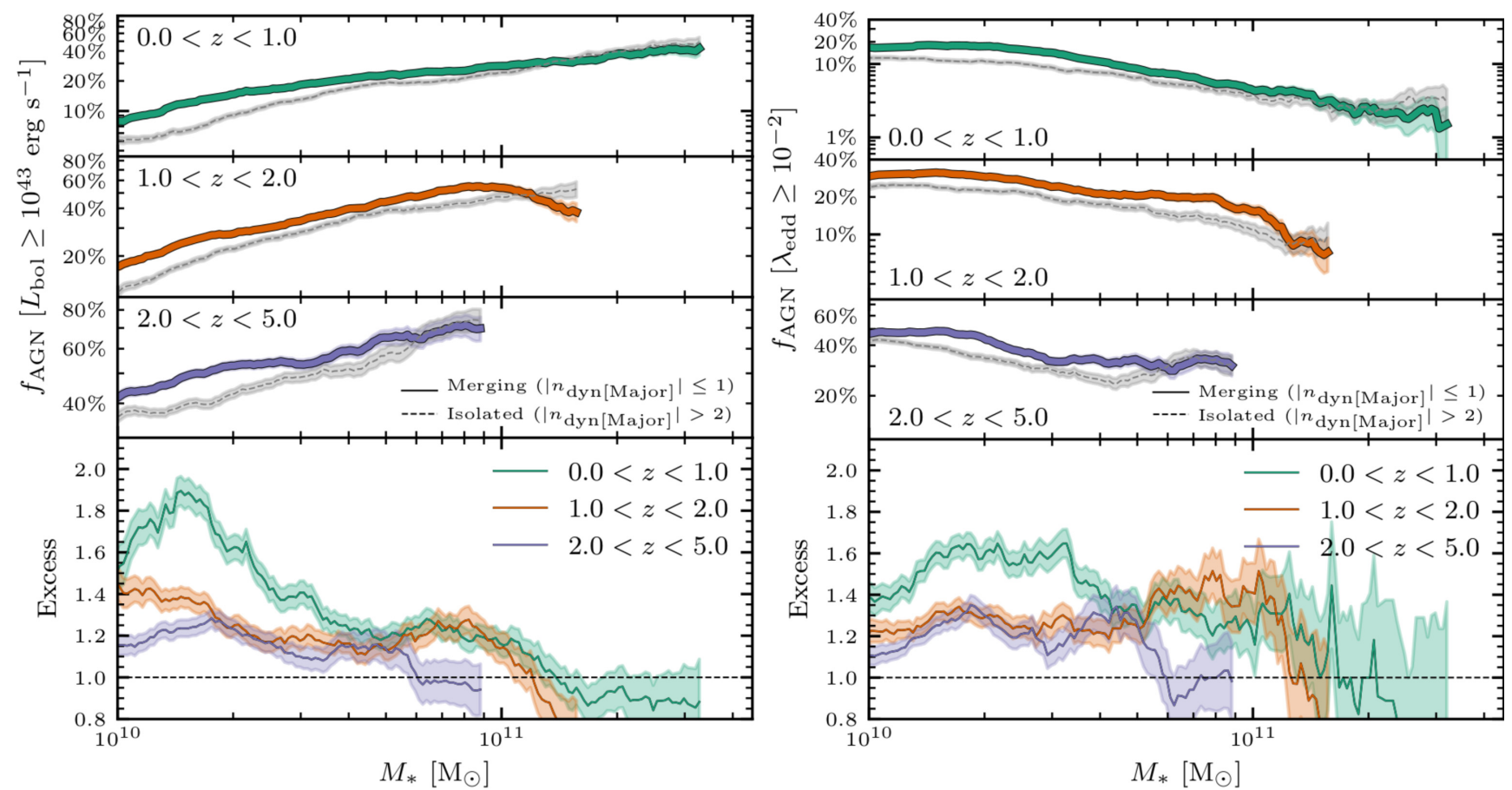

Figure 2. The AGN fraction of major mergers $\left(M_{*, 1} / M_{*, 2} \geq \frac{1}{4}\right)$ as a function of the stellar mass. We classify a galaxy as hosting an AGN if the BH has a bolometric AGN luminosity greater than $L_{\text {bol }} \geq 10^{43} \mathrm{erg} \mathrm{s}^{-1}$ (left-hand panel) or an Eddington rate greater than $\lambda_{\text {edd }} \geq 10^{-2}$ (right-hand panel). The AGN fraction of the associated samples of isolated galaxies acts as our control (see Section 2.3.1). The shaded regions indicate the Poisson uncertainty. As the stellar mass increases, the AGN fraction increases when defined by a cut in AGN luminosity and decreases when defined by a cut in Eddington rate. These trends are due to an increasing BH mass with increasing stellar mass, and are emulated in the trends of the isolated control galaxies. The excess between the AGN fraction of merging systems relative to the isolated control galaxies is shown in the lower panels: at lower redshifts $(z<1)$ the excess increases with decreasing stellar mass (up to a value of $\approx 1.8$ at $M_{*} \approx 10^{10} \mathrm{M}_{\odot}$ ), at higher redshifts $(z>1)$ the excess values maintain an approximately constant value of $\approx 1.1-1.3$ for all stellar masses. However, the spread at higher stellar masses $\left(M_{*} \gtrsim 10^{11} \mathrm{M}_{\odot}\right)$ are sufficiently large as to be consistent with no excess (see also Fig. 4).

pair separation is due to an increasing mean stellar mass and gas

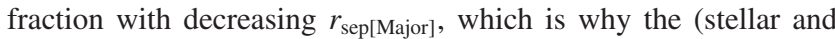
gas mass matched) control galaxies trace the trends so closely. This is caused by the fact that many of the close pair galaxies at larger separations $\left(r_{\mathrm{sep} \text { [Major] }} \gtrsim 50 \mathrm{pkpc}\right)$ are gas-poor satellite galaxies hosted within larger haloes $\left(M_{200} \sim 10^{13} \mathrm{M}_{\odot}\right)$, whereas at smaller separations $\left(r_{\text {sep[Major] }} \lesssim 50 \mathrm{pkpc}\right.$ ) the sample begins to become increasingly dominated by interactions between the central galaxies of lower mass haloes $\left(M_{200} \sim 10^{12} \mathrm{M}_{\odot}\right)$.

The right-hand panel of Fig. 3 repeats this analysis for when an AGN is defined by a cut in the Eddington rate $\left(\lambda_{\text {edd }} \geq 10^{-2}\right)$. Again, a weak trend of an increasing AGN fraction with decreasing pair separation is found: rising from $\approx 8 \pm 0$ per cent $\rightarrow 12 \pm 1$ per cent in the redshift range $0<z<1, \approx 19 \pm 1$ per cent $\rightarrow 25 \pm 1$ per cent in the redshift range $1<z<2$, and $\approx 34 \pm 2$ percent $\rightarrow$ $33 \pm 2$ percent in the redshift range $2<z<5$ (each reported for the $3 \mathrm{D}$ separation range $100 \geq r_{\mathrm{sep}[\text { Major] }} \geq 10 \mathrm{pkpc}$ ). The excess in the AGN fraction between the merging and isolated galaxies is much more prominent when the Eddington rate is considered: initially appearing at separations of $50 \lesssim r_{\mathrm{sep} \text { [Major }]} \lesssim 100 \mathrm{pkpc}$ and rising to an excess value of $\approx 1.1-1.3$ at $r_{\text {sep[Major] }} \approx 10 \mathrm{pkpc}$ (for redshifts $z<2)$. At higher redshifts $(z>2)$ there is little evidence for any enhancement in the AGN fractions when considering either the bolometric luminosity or the Eddington rate, however we note that the number of galaxies with stellar masses greater than $M_{*}>$ $10^{10} \mathrm{M}_{\odot}$ that have a major companion at close separations $\left(r_{\text {sep[Major] }}\right.$ $\lesssim 30 \mathrm{pkpc}$ ) are very limited within the simulation volume at these redshifts.
One could argue that the reduced values of the excess in the AGN fractions seen in Fig. 3 (particularly for the AGN luminosity) are in tension with the results from Figs 1 and 2. However, we remind the reader that the close pairs sample is only able to probe galaxies in a pre-merger stage when the two galaxies remain separated, whereas the other three samples additionally include galaxies in a postmerger stage (i.e. any triggered AGN activity post-coalescence is not seen in the close pair analysis, see also Section 3.3). We additionally note that greater excess values are seen between the AGN fraction of close pair galaxies and their isolated control galaxies if we consider a higher cut in the luminosity or Eddington rate to define an AGN (see Fig. A3).

Thus each of the three methods of analyses used in Sections 3.1.1 to 3.1.3 have reported a similar picture, that there exists a measurable excess of AGN activity during the course of a major merger.

\subsection{The optimal galaxies for enhancing $\mathrm{BH}$ activity during a major merger}

In the previous section we investigated the merger and AGN fractions for all galaxies more massive than $M_{*} \geq 10^{10} \mathrm{M}_{\odot}$, discovering a measurable enhancement of $\mathrm{BH}$ activity directly connected to the triggering influence of major mergers. To explore this enhancement in more depth, we now test under what conditions the triggering of $\mathrm{BH}$ activity during the course of a major merger is optimal. Here we only directly report the results for galaxies within the redshift range $0<z<1$ (where we have the greatest dynamic range of galaxy 

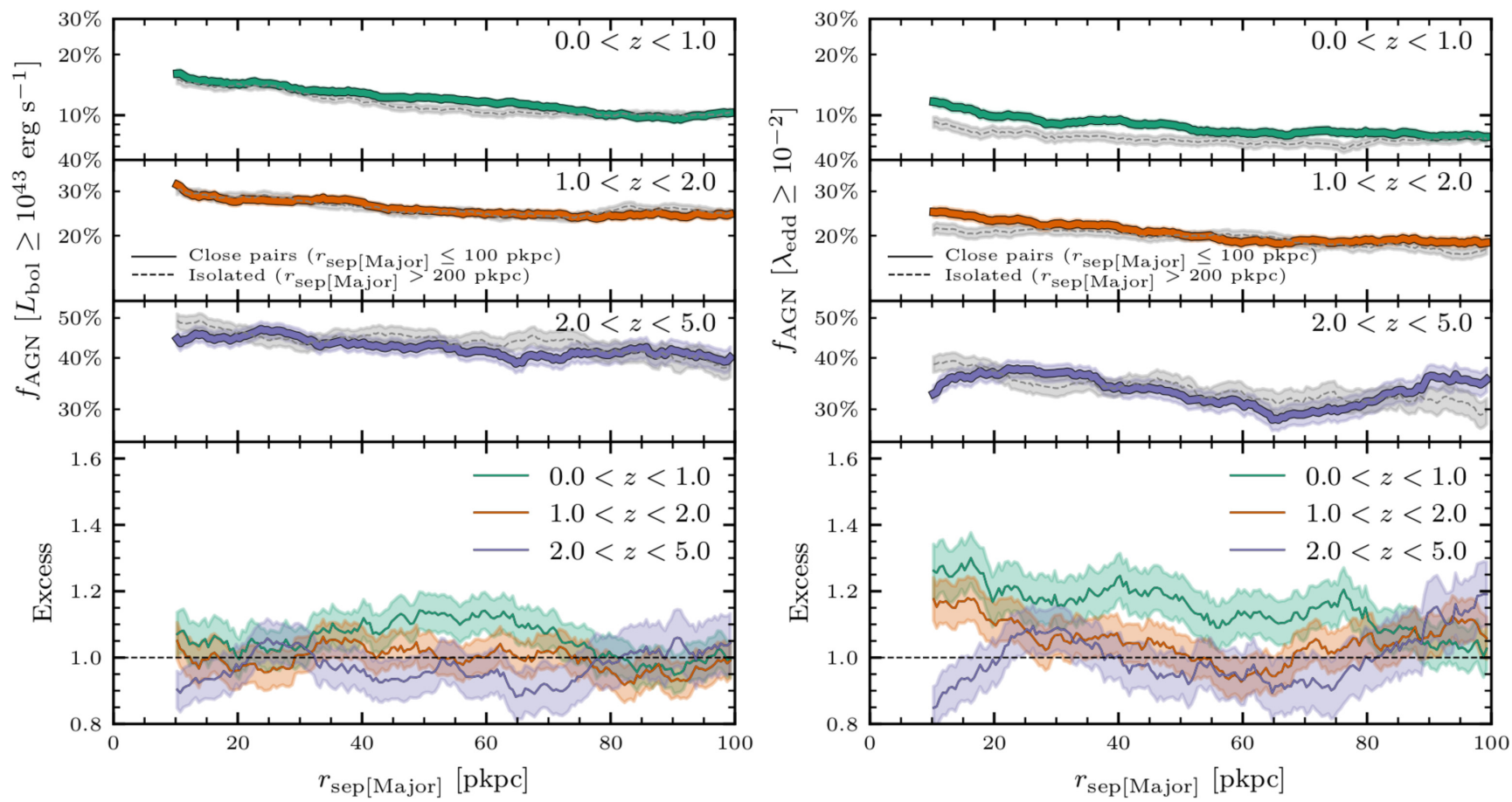

Figure 3. The AGN fraction of galaxies with a close major companion $\left(M_{*, 1} / M_{*, 2} \geq \frac{1}{4}\right)$ as a function of the 3D pair separation. We define a galaxy to be an AGN if it hosts a BH with a bolometric luminosity greater than $L_{\mathrm{bol}} \geq 10^{43} \mathrm{erg} \mathrm{s}^{-1}$ (left-hand panel) or an Eddington rate greater than $\lambda_{\text {edd }} \geq 10^{-2}$ (right-hand panel). The AGN fraction of the associated control sample of isolated galaxies is also shown (see Section 2.3.1). We note that the isolated control galaxies are linked to the galaxies within the close pairs sample using our matching criteria (see Section 2.3.1), and are presented on the figures using the 3D separation of the close pair galaxy (whilst themselves having no close major companions within $200 \mathrm{pkpc}$ ). The shaded regions indicate the Poisson uncertainty. When the AGN fraction is defined by either a cut in the bolometric luminosity or the Eddington rate, there is a weak trend of a rising AGN fraction with decreasing pair separation. The excess in the AGN fraction of close pair galaxies relative to the isolated control galaxies is shown in the lower panels. For the AGN luminosity in the left-hand panel, there is a hint that an excess first appears at separations of $r_{\mathrm{sep}[\mathrm{Major}]} \approx 80 \mathrm{pkpc}$, and potentially increases towards lower separations up to a maximum value of $\approx 1.1$ (yet the errors mean the excess is often consistent with 1 , i.e. no excess). However, when an AGN is defined by a cut in the Eddington rate in the right-hand panel, an increasing excess value with decreasing pair separation is much more prominent: starting at $50 \lesssim r_{\text {sep[Major] }} \lesssim$ $100 \mathrm{pkpc}$ and reaching a peak excess of $\approx 1.1-1.3$ at $\approx 10 \mathrm{pkpc}$ (for $z<2$ ).

properties), however we note that the behaviours at higher redshifts are very similar.

In Fig. 2 we found an increasing excess in the number of AGN that reside in merging galaxies, relative to the associated control sample of isolated galaxies, with decreasing stellar mass (most strongly at redshifts $0<z<1$ ). Because of this, we first revisit the results of Figs 1 and 3, to see if there exists a similar stellar mass dependence upon the excess merger and AGN fractions reported in the lower panels.

The upper panel of Fig. 4 shows the excess of the merger fraction between active $\left(L_{\mathrm{bol}} \geq 10^{43} \mathrm{erg} \mathrm{s}^{-1}\right)$ and inactive BHs $\left(L_{\mathrm{bol}}\right.$ $<10^{43} \mathrm{erg} \mathrm{s}^{-1}$ ) as a function of the bolometric AGN luminosity (i.e. the lower left-hand panel of Fig. 1), with the galaxies now subdivided into three stellar mass ranges. It is immediately clear that major mergers do not uniformly enhance $\mathrm{BH}$ activity across all of the galaxies within the sample: the BHs hosted by lower mass galaxies $\left(1 \times 10^{10}<M_{*}<5 \times 10^{10} \mathrm{M}_{\odot}\right)$ show the greatest enhancement of $\mathrm{BH}$ activity over their inactive counterparts (reaching excess values of $\approx 3$ at $\approx 1 \times 10^{45} \mathrm{erg} \mathrm{s}^{-1}$, over twice the excess that was seen in Fig. 1), and the most massive galaxies $\left(M_{*} \gtrsim 10^{11} \mathrm{M}_{\odot}\right)$ show essentially no enhancement in BH activity over their inactive counterparts. This echoes the results from Fig. 2, where the excess in the AGN fraction of merging galaxies over their isolated counterparts was mostly restricted to lower mass systems $\left(M_{*} \sim 10^{10} \mathrm{M}_{\odot}\right)$. The lower panel of Fig. 4 repeats this analysis for the excess of the merger fraction between active $\left(\lambda_{\text {edd }} \geq 10^{-2}\right)$ and inactive BHs $\left(\lambda_{\text {edd }}<10^{-2}\right)$ as a function of the Eddington rate (i.e. the lower right-hand panel of Fig. 1), finding that the largest excess values, and the BHs with the highest Eddington rates, are again almost exclusively found in lower mass systems $\left(M_{*} \lesssim\right.$ $\left.5 \times 10^{10} \mathrm{M}_{\odot}\right)$.

In a similar manner, Fig. 5 returns to the analysis of Fig. 3, investigating the excess between the AGN fractions of galaxies with close major companions and isolated galaxies as a function of the pair separation, now in three bins of stellar mass (we note there are too few galaxies above $M_{*} \geq 10^{11} \mathrm{M}_{\odot}$ within the close pair sample to retrieve meaningful statistics when the Eddington rate is considered). The upper panel of Fig. 5 classifies an AGN by a cut in the bolometric AGN luminosity $\left(L_{\mathrm{bol}} \geq 10^{43} \mathrm{erg} \mathrm{s}^{-1}\right.$; i.e. the lower left-hand panel of Fig. 3) and the lower panel of Fig. 5 classifies an AGN by a cut in the Eddington rate $\left(\lambda_{\text {edd }} \geq 10^{-2}\right.$; i.e. the lower right-hand panel of Fig. 3). Whilst not as elevated as the excess values in Fig. 4, we similarly find that less massive systems with close major companions are the ones with the largest excess in their AGN fractions over their isolated counterparts, and, again, the most massive galaxies $\left(M_{*} \gtrsim 5 \times 10^{10} \mathrm{M}_{\odot}\right)$ show little evidence for any enhancement in their AGN fractions over their isolated counterparts.

In addition to the stellar mass, investigating further properties of galaxies may continue to refine what are the optimal conditions for 

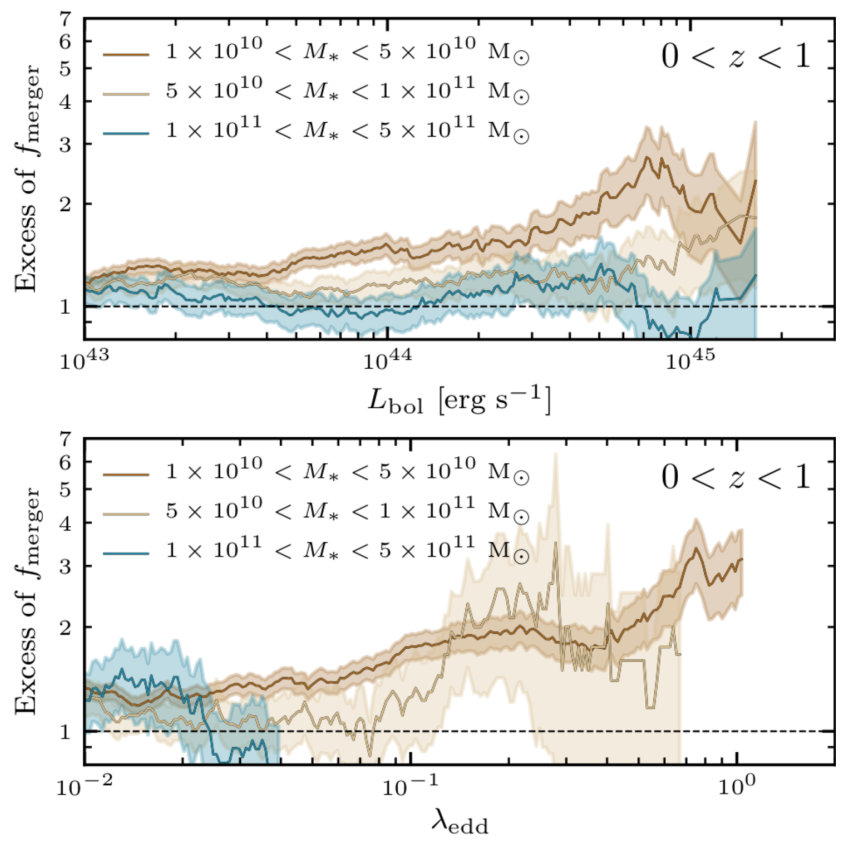

Figure 4. The excess in the major merger fraction from the lower panels of Fig. 1, with the galaxies in the redshift range $0<z<1$ now subdivided into three stellar mass ranges. Both when investigated as a function of the AGN luminosity (upper panel), or as a function of the Eddington rate (lower panel), the excess in the merger fraction comes almost exclusively from lower mass galaxies $\left(M_{*} \lesssim 5 \times 10^{10} \mathrm{M}_{\odot}\right)$. Higher mass galaxies $\left(M_{*} \gtrsim 10^{11} \mathrm{M}_{\odot}\right)$ show no excess in their merger fractions between active and inactive galaxies. This suggests that the enhancement of $\mathrm{BH}$ activity triggered via a major merger is restricted to less massive systems $\left(M_{*} \lesssim 10^{11} \mathrm{M}_{\odot}\right)$.

triggering $\mathrm{BH}$ activity during a major merger. Fig. 6 again shows the excess of the major merger fraction from the lower left-hand panel of Fig. 1, with the galaxies now subdivided into ranges of the total gas fraction $\left(f_{\text {gas }} \equiv \frac{M_{\text {gas }}}{M_{\text {gas }}+M_{*}}\right.$, upper panel), the BH mass (middle panel), and distinguishing between central and satellite galaxies (lower panel). Intuitively, the merging galaxies with the highest gas fractions $\left(f_{\text {gas }}>0.2\right)$ show the greatest excess values in their merger fractions above their inactive counterparts. In addition, we find that the galaxies hosting less massive $\mathrm{BHs}\left(M_{\mathrm{BH}}<10^{7} \mathrm{M}_{\odot}\right)$ display the greatest excess values, in line with the picture that less massive galaxies are those with the highest excess values (see Figs 4 and 5). Finally, central galaxies appear responsible for much of the excess, as opposed to gas-poor satellite galaxies, particularly at higher AGN luminosities.

It is not entirely clear why $\mathrm{BH}$ activity triggered via a merger should be restricted to galaxies of lower masses $\left(M_{*} \lesssim 10^{11} \mathrm{M}_{\odot}\right)$. The simplest explanation is that for a galaxy to sustain an AGN for a period of time it requires an adequate supply of fuel (i.e. a high gas fraction), most commonly present in lower mass galaxies. Additionally, the higher mass BHs occupying higher mass galaxies could more rapidly extinguish continued accretion over their lower mass counterparts via efficient AGN feedback (given the larger accretion rates achieved by more massive BHs under the same surrounding gas conditions, see equation 2).

Thus the results from Sections 3.1 and 3.2 have shown that major mergers do trigger an increased amount of AGN activity within the EAGLE universe, and that it is most measurable at the highest AGN luminosities $\left(L_{\text {bol }} \sim 10^{45} \mathrm{erg} \mathrm{s}^{-1}\right)$ and Eddington rates $\left(\lambda_{\text {edd }} \approx 1\right)$,
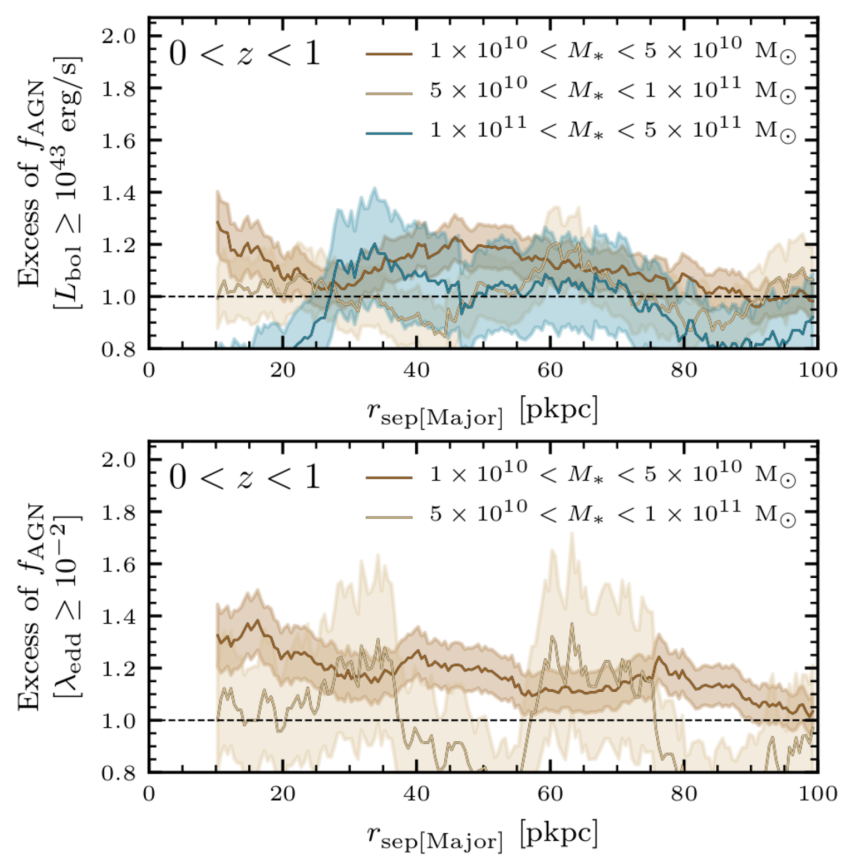

Figure 5. The excess in the AGN fraction from the lower panels of Fig. 3, with the galaxies in the redshift range $0<z<1$ now subdivided into three stellar mass ranges. When an AGN is classified by either a cut in the bolometric AGN luminosity ( $L_{\mathrm{bol}} \geq 10^{43} \mathrm{erg} \mathrm{s}^{-1}$, upper panel), or by a cut in the Eddington rate $\left(\lambda_{\text {edd }} \geq 10^{-2}\right.$, lower panel), the largest enhancement in the AGN fraction is found in lower mass systems $\left(M_{*} \lesssim 5 \times 10^{10} \mathrm{M}_{\odot}\right)$. Higher mass galaxies $\left(M_{*} \gtrsim 1 \times 10^{11} \mathrm{M}_{\odot}\right)$ with close major companions show no sign of any excess in their AGN fractions over their isolated counterparts. This suggests that the enhancement of $\mathrm{BH}$ activity triggered via a major merger is restricted to less massive systems $\left(M_{*} \lesssim 10^{11} \mathrm{M}_{\odot}\right)$.

within lower mass central galaxies $\left(M_{*} \lesssim 10^{11} \mathrm{M}_{\odot}\right)$ with higher gas fractions $\left(f_{\text {gas }}>0.2\right)$ that host lower mass BHs $\left(M_{\mathrm{BH}} \sim 10^{6} \mathrm{M}_{\odot}\right)$ at lower redshifts $(z<1)$.

\subsection{The enhancement of BH activity during different stages of a major merger}

For the analysis in Section 3.1 we only considered our fiducial definition of a merging system: a galaxy is in the state of a merger if it has completed or will complete a major merger within \pm 1 dynamical time (see Section 2.4). However such a broad time window will shield the relative importance of each merger stage for enhancing $\mathrm{BH}$ activity (e.g. the interacting, coalescence, and remnant phases). To explore this, we now investigate the AGN fraction of galaxies at various stages of a major merger (parametrized by the number of dynamical times to the coalescence of the two galaxies, i.e. $\left.n_{\text {dyn[Major] }}\right)$, to see when, if at all, an optimal stage for triggering $\mathrm{BH}$ activity exists. Here we use the galaxies from the 'Major mergers' sample (see Table 1).

Fig. 7 shows the AGN fraction of galaxies at five predefined stages of a major merger, starting from the initial interaction through to the final remnant. We categorize each merger stage using a fixed window of $n_{\text {dyn[Major] }}$, i.e. a fixed window of the number of dynamical times to the coalescence of the two galaxies: 'early interacting' $\equiv$ $-1.0<n_{\text {dyn[Major }]}<-0.5$, 'late interacting' $\equiv-0.5<n_{\text {dyn[Major }]}<$ -0.1 , 'coalescence' $\equiv-0.1<n_{\text {dyn[Major }]}<0.1$, 'early remnant' $\equiv$ 


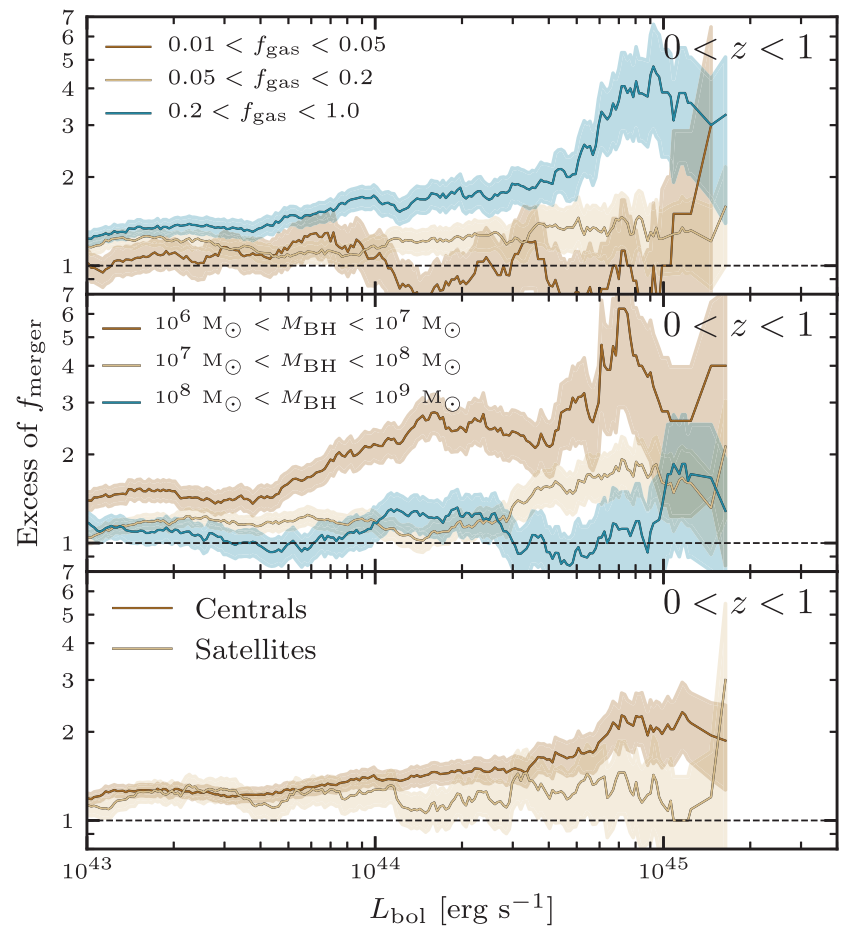

Figure 6. The excess in the major merger fraction from the lower lefthand panel of Fig. 1, with the galaxies in the redshift range $0<z<1$ now subdivided into ranges of the total gas fraction ( $f_{\text {gas }} \equiv \frac{M_{\text {gas }}}{M_{\text {gas }}+M_{*}}$, upper panel), the $\mathrm{BH}$ mass (middle panel) and distinguishing between central and satellite galaxies (lower panel). We find the largest excess between the merger fraction of AGN and inactive galaxies comes from the galaxies that are the most gas rich $\left(f_{\text {gas }}>0.2\right)$, those that host lower mass BHs $\left(M_{\mathrm{BH}} \sim\right.$ $10^{6} \mathrm{M}_{\odot}$ ), and from those that are central galaxies.

$0.1<n_{\text {dyn[Major }]}<0.5$, and 'late remnant' $\equiv 0.5<n_{\text {dyn[Major }]}<1.0 .^{6}$ That is, we redefine the values of $a$ and $b$ in equation (5) to these new limits. The AGN fraction of the matched isolated control galaxies associated with the merging galaxies at each stage is also shown. We find, for each redshift range, that the AGN fraction is not constant throughout the merger process, and instead slowly rises and declines throughout the course of the interaction, peaking during the early remnant phase. This tells us that the greatest abundance of AGN during a major merger are found soon after the two galaxies have already coalesced. If we then compare the AGN fractions of the merging systems to the isolated control galaxies, we also find that the greatest enhancement of AGN is during the early remnant stage (most notably in the lower two redshift ranges, $z<2$ ).

Taking this investigation further, Fig. 8 shows, now purely as a function of the number of dynamical times to coalescence (i.e. no predefined phases), the excess in the AGN fraction of galaxies at a particular stage in a major merger relative to the AGN fraction of their associated isolated control galaxies. As a reminder: negative values of $n_{\text {dyn[Major] }}$ indicate the nearest major merger is in the future and the system is still in an interacting/pre-coalescence

${ }^{6}$ For galaxies at $z=0.5$ these time dynamical time windows correspond to cosmic time windows of: 'early interacting' $\equiv-1.22<t-t_{\text {merger }}$ $<-0.61 \mathrm{Gyr}$, 'late interacting' $\equiv-0.61<t-t_{\text {merger }}<-0.12 \mathrm{Gyr}$, 'coalescence' $\equiv-0.12<t-t_{\text {merger }}<0.12 \mathrm{Gyr}$, 'early remnant' $\equiv 0.12<$ $t-t_{\text {merger }}<0.61 \mathrm{Gyr}$ and 'late remnant' $\equiv 0.61<t-t_{\text {merger }}<1.22 \mathrm{Gyr}$. At higher and lower redshifts the dynamical time windows will correspond to shorter and longer cosmic time windows, respectively (see equation 3).

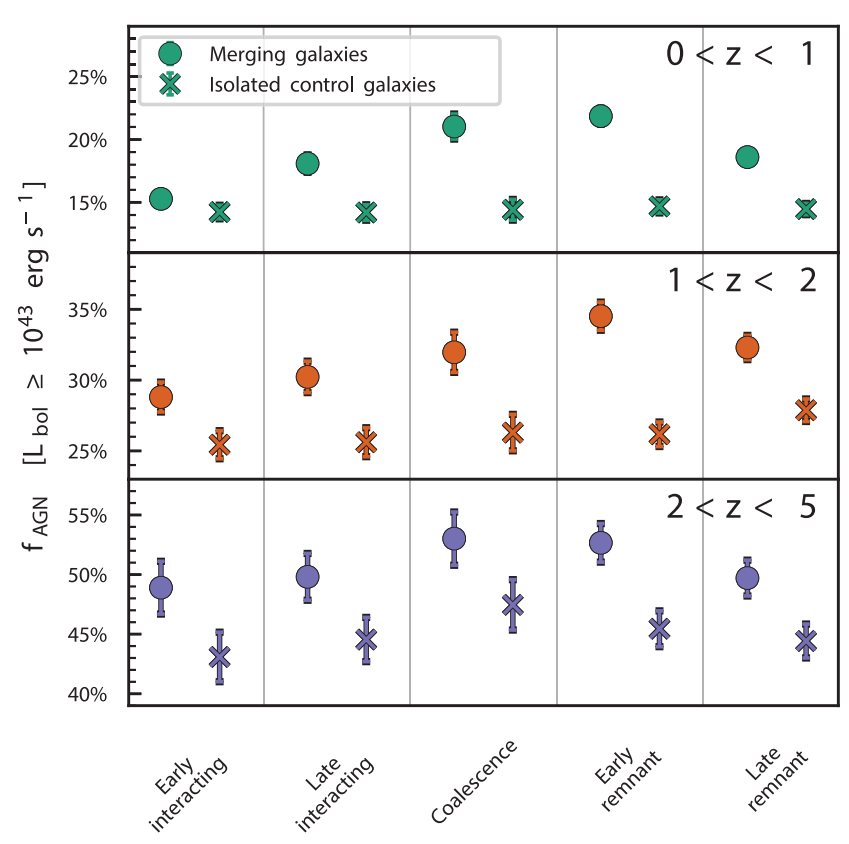

Figure 7. The AGN fraction of galaxies at five predefined stages of a major merger. Each stage is defined using a fixed window of $n_{\text {dyn[Major] }}$, i.e. a fixed window of the number of dynamical times to the coalescence of the two galaxies (see Section 3.3). At each redshift the AGN fraction rises from the early interacting stage to the early remnant phase, and declines towards the late remnant phase. To compare, the AGN fraction of the matched sample of isolated control galaxies is also shown, revealing that there is typically an increased amount of $\mathrm{BH}$ activity at most stages of a major merger relative to their isolated counterparts. The greatest excess of AGN activity during a merger is during the early remnant phase (i.e. after the two galaxies have already coalesced, see also Fig. 8). The error bars indicate the Poisson uncertainty.

phase, positive values of $n_{\text {dyn[Major] }}$ indicate the nearest major merger occurred in the past and the system is in a remnant/post-coalescence phase, and values very close to zero indicate the system is in the final stages of coalescence. In the upper panel a galaxy is defined to be active if it has a bolometric AGN luminosity greater than $L_{\mathrm{bol}}$ $\geq 10^{43} \mathrm{erg} \mathrm{s}^{-1}$ (the same as Fig. 7), and in the lower panel a galaxy is defined to be active if it has an Eddington rate greater than $\lambda_{\text {edd }}$ $\geq 10^{-2}$.

For the higher two redshift ranges $(z>1)$, and for both definitions of an active $\mathrm{BH}\left(L_{\mathrm{bol}} \geq 10^{43} \mathrm{erg} \mathrm{s}^{-1}\right.$ or $\left.\lambda_{\text {edd }} \geq 10^{-2}\right)$, an excess in the AGN fraction first appears $\approx 1$ dynamical time $(\approx 1.2 \mathrm{Gyr}$ at $z=0.5)$ before the coalescence of the two galaxies, oscillates steadily around excess values of 1.2-1.3 until 1 dynamical time after the coalescence of the two galaxies, and then continues to decline towards higher values of $n_{\text {dyn[Major] }}$. If we integrate under the curve between the limits $-1<n_{\text {dyn[Major] }}<1$ (i.e. our definition of a merging state) we find a very similar total excess both before and after the coalescence of the two galaxies. This means that $\approx 50$ percent of the excess values at $z>1$ reported in Figs 1 and 2 originate from the remnants of merging galaxies. The behaviour changes somewhat at lower redshifts $(z<1)$, now with the majority of enhanced $\mathrm{BH}$ activity triggered via the merging process occurring after the coalescence of the two galaxies ( 65 per cent and 75 per cent in the upper and lower panels, respectively, again in the limits $-1<n_{\text {dyn[Major] }}<1$ ). This means that at lower redshifts a significant majority of the excess values reported in Figs 1 and 2 originate from the remnants of merging galaxies. In addition, the distribution at lower redshifts is 


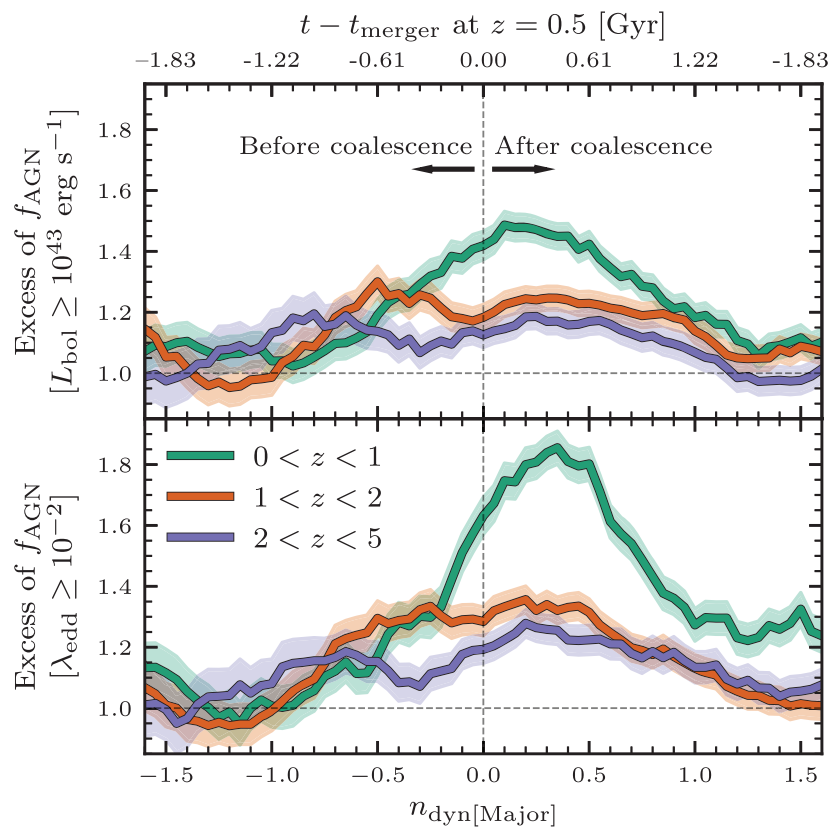

Figure 8. The excess in the AGN fraction (defined by a cut in the luminosity in the upper panel and by a cut in Eddington rate in the lower panel) at each stage of a major merger (parametrized by the number of dynamical times to the coalescence of the two galaxies, i.e. $n_{\mathrm{dyn}[\mathrm{Major}]}$ ) relative to the AGN fraction of the associated control sample of isolated galaxies. Here we are showing when during a major merger $\mathrm{BH}$ activity is most enhanced. Positive values of $n_{\text {dyn[Major] }}$ indicate the system is post-coalescence, negative values of $n_{\text {dyn[Major] }}$ indicate the system is pre-coalescence and $n_{\text {dyn[Major] values }}$ of $\approx 0$ indicate the system is in the final stages of coalescence. At higher redshifts $(z>1)$, an excess in the AGN fraction first appears $\approx 1$ dynamical time before the coalescence of the two galaxies, maintains a value of 1.2 1.3 until $\approx 1$ dynamical time after the coalescence of the two galaxies, and

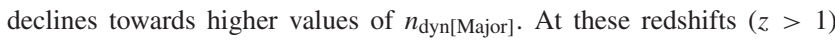
the total excess in the AGN fraction originates from galaxies both before and after coalescence, with an approximately equal weighting (50/50). At lower redshifts $(z<1)$, the majority of the excess in the AGN fraction originates from galaxies post-coalescence, and the distribution is peaked around $n_{\text {dyn[Major] }} \approx 0.25$, which corresponds to $\approx 300$ Myr of cosmic time at $z=0.5$. This indicates that a significant fraction $(\approx 65-75$ per cent $)$ of $\mathrm{BH}$ activity that is triggered via a merger occurs within the remnants of merging systems at $z<1$.

distinctly peaked around a value of $n_{\text {dyn[Major] }} \approx 0.25$, corresponding to $\approx 300$ Myr of cosmic time at $z=0.5$, suggesting there is typically a significant delay between the coalescence of the two galaxy nuclei and triggered $\mathrm{BH}$ activity at $z<1$.

\section{DISCUSSION}

\subsection{The effect of the model}

When analysing the results from cosmological hydrodynamical simulations, such as EAGLE, it is always important to consider how the adopted subgrid models may influence the interpretation of the results. For this study, the most relevant subgrid models are those that govern the behaviour of BHs, which we briefly described in Section 2.1, and are fully described in Schaye et al. (2015).

The accretion rate of $\mathrm{BHs}$ in the simulation is directly proportional to the density of the surrounding gas, and the square of the mass of the $\mathrm{BH}\left(\dot{m}_{\mathrm{BH}} \propto m_{\mathrm{BH}}^{2} \rho\right.$, see equation 2$)$. Thus a high accretion rate can be created as the density of the surrounding gas increases, for example as it is funnelled inward or compressed during the course of a merger, or simply by having a massive $\mathrm{BH}$. Each of these two routes can readily produce visibly 'active' BHs, and both contribute to the upward trends found in Figs 1 and 2. These effects are not necessarily contentious, as we would expect better fuelled and larger BHs to be increasingly capable of producing more luminous AGN. However, given that both a jump in the surrounding gas density during a merger versus there simply being an already massive $\mathrm{BH}$ are degenerate to the eventual accretion rate, it is not always straightforward to decouple the dominant contributor to any increased AGN activity.

The dependence between the accretion rate of the $\mathrm{BH}$ and the square of the $\mathrm{BH}$ mass will, at least in part, be responsible for the increased excess of AGN activity seen after the coalescence of the two galaxies has completed (see Figs 7 and 8). This results from the fact that as the two BHs eventually coalesce (following the coalescence of the two galaxies), the sudden jump in BH mass will result in an even greater jump in the accretion rate (assuming the same conditions of the surrounding gas), increasing the likelihood for a 'visible' AGN in the merger remnant. Additionally, the characteristic time-scale between the two galaxies coalescing and the eventual coalescence of the two BHs is dependent on the $\mathrm{BH}$ merging criteria adopted by the simulation. For EAGLE, the coalescence of two $\mathrm{BHs}$ is not a resolved process, and we therefore implement broad conditions for this process to occur: the two BHs must be within each others smoothing kernel and their relative velocity to one another must be less than the circular velocity at that distance. It is likely that these conditions merge the BHs earlier than they should (e.g. Rantala et al. 2017), which would potentially result in a rightward shifting of the excess peaks in Fig. 8 (i.e. the peak of AGN activity would lag further behind the coalescence of the two galaxies). However, we do not anticipate any of the overall behaviour or trends of this study would be affected by this, with the majority of the triggered AGN activity still occurring post-coalescence (of the galaxy nuclei).

\subsection{Comparing to observations}

Both at lower and higher redshifts, as of yet there remains no unanimous consensus as to the importance of galaxy-galaxy mergers for triggering $\mathrm{BH}$ activity from observational data. A possible exception is the most luminous $\left(L_{\mathrm{bol}} \gtrsim 10^{46} \mathrm{erg} \mathrm{s}^{-1}\right)$, typically heavily obscured quasars, which are found to reside almost exclusively in disturbed systems, suggesting a merger driven scenario at least in this regime (e.g. Glikman et al. 2015; Fan et al. 2016). However one should be careful on how to interpret systems with such strong selection biases. Yet even amongst the uncertainty that has arisen between the empirical results, it is still informative to compare the results of the simulation to the observations where possible, along with making predictions for future observations.

For this study we have deliberately chosen to avoid a quantitative comparison with observations when a 'merging state' has to be defined, such as for the results in Sections 3.1.1 and 3.1.2. In the simulation we have the advantage of knowing when two galaxies will, or have, coalesced, which we parametrized by the number of dynamical times to that event. However observational works must ascertain the merging state of a galaxy from only an instantaneous (often pre-coalescence) snapshot. Thus a truly fair comparison would require us to apply observational techniques to synthetic images to estimate a galaxy's current merging state (similar to Lahén et al. 2018; Bottrell et al. 2019; Snyder et al. 2019, for example), but 
this is beyond the scope of this study. We can, however, qualitatively compare our results to the observational studies.

The trend of an increasing merger fraction with increasing AGN luminosity, similar to the trends found in Fig. 1, has been discovered empirically (e.g. Ellison et al. 2019). More broadly, the observed fraction of merging galaxies that host an AGN, or the fraction of AGN found to reside in merging systems, are often reported to be higher than the samples of inactive or isolated control galaxy counterparts (e.g. Koss et al. 2010; Ellison et al. 2011; Rosario et al. 2015; Goulding et al. 2018). These observations therefore agree with the results presented in Figs 1 and 2, and suggests, both in the observations and the simulation, that mergers are directly responsible for triggering an increased amount of $\mathrm{BH}$ activity for at least a subset of the galaxy population. However, these observational results, and therefore our own, then disagree with the empirical studies that find no discernible enhancement in AGN activity around the time of a merger (e.g. Kocevski et al. 2012; Schawinski et al. 2015; Villforth et al. 2017; Marian et al. 2019). When it comes to the observed AGN fraction of galaxies with close companions, an increasing excess of AGN with decreasing pair separation has been found (e.g. Ellison et al. 2011; Silverman et al. 2011), further reinforcing the mergers triggering $\mathrm{BH}$ activity scenario, and again agreeing with the results from this study (see Fig. 3 and also Section 4.2.1). Thus qualitatively the results presented by this study are in good agreement with many current observational works that have investigated the merger-AGN connection.

For future observations, we predict that the strongest observable signal connecting enhanced $\mathrm{BH}$ activity to galaxy-galaxy mergers will come more from high Eddington rate sources, as opposed to high luminosity sources (such as was done in Marian et al. 2019). In addition, we predict that the excess in the merger and AGN fractions will be greatest at lower redshifts (i.e. $z<1$ ), and the galaxies exhibiting the most optimal conditions for triggering an AGN via a merger are those with lower masses $\left(M_{*} \sim 10^{10} \mathrm{M}_{\odot}\right)$, higher gas fractions $\left(f_{\text {gas }} \geq 0.2\right)$ and lower mass BHs $\left(M_{\mathrm{BH}} \sim 10^{7} \mathrm{M}_{\odot}\right.$; see Figs 2 and 4 to 6 ). For the three methods of analysis used in Section 3, we consistently found no enhancement of BH activity during the period of a merger in the most massive galaxies $\left(M_{*} \gtrsim\right.$ $10^{11} \mathrm{M}_{\odot}$ ), relative to their inactive or isolated counterparts (where some observational studies have reported their strongest signals of AGN enhancement; e.g. Goulding et al. 2018).

A key finding of this study was discovering that 50-75 per cent of enhanced $\mathrm{BH}$ activity triggered by major mergers comes after the two galaxies have already coalesced (see Figs 7 and 8). Indeed, a much weaker excess was found in the AGN fraction over their isolated counterparts if we restricted our sample to just the galaxies currently in their interacting stages (see Fig. 3), which is the stage where the majority of observational samples will be capturing galaxies in the state of a merger. It is therefore crucial that observational studies are able to robustly identify post-merger remnants, so as to not mistakenly classify these AGN as being hosted by isolated systems. Encouragingly, studies have shown that post-merger features could have observability time-scales of $\approx 0.2-$ 0.4 Gyr (e.g. Lotz et al. 2010; Ji, Peirani \& Yi 2014), which would mean that the peak excess of $\mathrm{BH}$ activity that arises $\approx 300 \mathrm{Myr}$ after the coalescence of the two galaxies found in Fig. 8 at $z<$ 1 could be captured, and therefore would be correctly attributed to a post-merger system. Indeed, some observational results have reported that the largest excess of AGN activity has been found in post-merger systems (e.g. Ellison et al. 2013; Koss et al. 2018), in agreement with the findings of this study.
As a final note, we investigated in Appendix A2 how the choice of matching criteria to select the control galaxies could affect the results of studies of this nature. For this study we matched the control galaxies using the redshift, stellar mass, halo mass, BH mass, gas mass, and the environment (through the $r_{2}$ and $N_{2}$ parameters), to ensure that the control galaxies were as similar as possible to the selected galaxies (see Section 2.3.1). However, these criteria cannot be trivially adopted for observations, with the majority opting to match on just the redshift and the stellar mass. Generally, we found that when fewer parameters are considered in the matching criteria, the excess values of both the merger fraction of AGN and the AGN fraction of merging systems (i.e. the lower left-hand panels of Figs 1 and 2) are typically higher (see Fig. A4). This could imply that observational studies that only match their control galaxies on the stellar mass and redshift are slightly overestimating their values of the excess fractions. However, the behaviours of the loosest control matching criteria are consistent with the strictest control matching criteria, and the excess values are never more than 50 percent different (and often much less, see Fig. A4). Larger differences are seen in the excess fractions when the Eddington rate is considered, varying by up to a factor of two in the excess values between the loosest and strictest matching criteria (see Fig. A4). This is because the control population becomes biased relative to the galaxies within the selected samples when matched on fewer parameters, caused by the fact that high Eddington rate AGN BHs are typically undermassive for galaxies of their stellar mass. It therefore appears that the excess values when considering the Eddington rate could be rather overestimated when the control galaxies are not matched on the BH mass. Finally, if the environment is not considered (through the $r_{2}$ and $N_{2}$ parameters) when investigating the AGN fraction of close pairs (i.e. Fig. 3), the AGN fraction of the control galaxies can be overestimated, resulting from gas-poor satellite galaxies of massive haloes getting mistakenly assigned to gas-rich central galaxies of the same mass. Yet overall, whilst it is recommended to match on as many parameters as possible, the behaviours recovered for each analysis method are largely unaffected by the choice of matching criteria, and the excess values are often well within 50 per cent of one another between the loosest and strictest matching criteria.

\subsubsection{Directly comparing to observations of the AGN fraction of close pairs}

To conclude this section, we examine how the results from the EAGLE simulation quantitatively compare to the observations of galaxies in the local Universe with close major companions taken from the Sloan Digital Sky Survey Data Release 7 (SDSS DR7). The observed galaxies are classified as hosting an AGN based on the cut of Kauffmann et al. (2003), with an S/N >3 required for all the requisite diagnostic emission lines. The SDSS sample consists of 7216 galaxies above a stellar mass of $M_{*} \geq 10^{10} \mathrm{M}_{\odot}$ in the redshift range $0.05<z<0.10$ that have a close major $\left(M_{*, 1} / M_{*, 2} \geq \frac{1}{4}\right)$ companion within a separation of 100 projected $\mathrm{kpc}$ and a relative velocity to within $\Delta v \leq 300 \mathrm{~km} \mathrm{~s}^{-1}$. For this analysis, the control galaxies from both the observations and the simulation are matched on redshift, stellar mass, and the environment (through the $r_{2}$ and $N_{2}$ parameters), using the same method and tolerance levels as outlined in Section 2.3.1. We note, that when applying the same selection to the simulation, the stellar mass distributions between the observed and simulated samples are not the same, with the simulated galaxy sample containing a greater proportion of lower mass galaxies $(M$ 


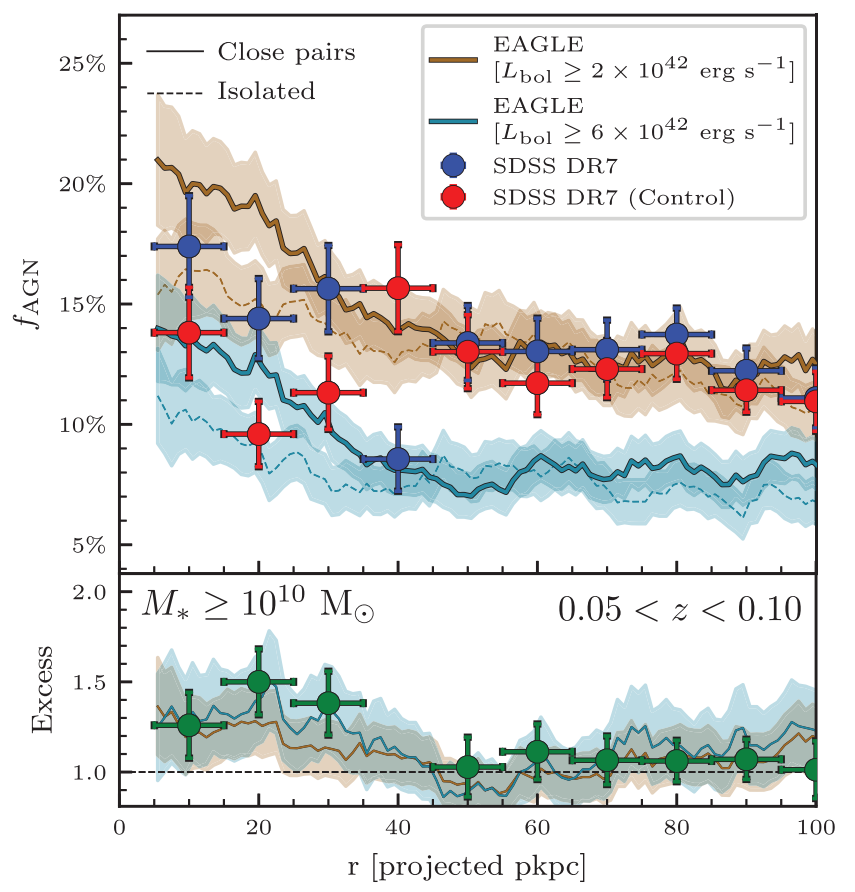

Figure 9. The AGN fraction of galaxies with close major companions $\left(M_{*, 1} / M_{*, 2} \geq \frac{1}{4}\right)$ as a function of the projected pair separation compared to observations taken from the Sloan Digital Sky Survey Data Release 7 (SDSS DR7). For both the simulation and the observations, the AGN fractions of the associated isolated control galaxies are also shown (see Section 2.3.1). For EAGLE, a BH is classified as 'active' if it has a bolometric AGN luminosity greater than either of the two quoted cuts. For the observed sample, a BH is classified as 'active' based on the cut of Kauffmann et al. (2003), with an $\mathrm{S} / \mathrm{N}>3$ required for all the requisite diagnostic emission lines. Only galaxies more massive than $M_{*} \geq 10^{10} \mathrm{M}_{\odot}$ in the redshift range $0.05<z<$ 0.10 are considered for each sample. There is an increasing AGN fraction with decreasing pair separation below $r \lesssim 40$ projected $\mathrm{kpc}$, and the excess between the AGN fraction of the close pair galaxies and their associated isolated control galaxies (shown in the lower panel) also only exists below these separations (up to an excess value of $\approx 1.5$, albeit with large errors).

$\sim 10^{10} \mathrm{M}_{\odot}$ ). To ensure that this does not have an impact on the results, we have rerun the analysis whereby we mass match the galaxies from the simulation to the observations in each bin of projected separation, indeed finding no significant change in the result.

The comparison is shown in Fig. 9, showing the AGN fraction of galaxies with close major companions as a function of the projected separation in the upper panel, and the excess between the AGN fraction of the close pair galaxies and the AGN fraction of their associated isolated control galaxies in the lower panel (analogous to Fig. 3). As we cannot classify if a galaxy hosts an AGN in the same manner as the observations, we perform the analysis with two cuts in the bolometric AGN luminosity to define an 'active' BH: $L_{\text {bol }} \geq 2 \times 10^{42} \mathrm{erg} \mathrm{s}^{-1}$ and $L_{\text {bol }} \geq 6 \times 10^{42} \mathrm{erg} \mathrm{s}^{-1}$. These cuts have been chosen to match the normalization of the observed AGN fractions, and also to demonstrate how sensitive the AGN fractions are to this choice. The behaviour of the simulation for both cuts of AGN luminosity are very similar, showing a rising AGN fraction with decreasing pair separation, only differing from one another by their overall normalization. This rising trend is also apparent in the observed sample, however the rise in the AGN fraction at smaller separations ( $r \lesssim 20$ projected $\mathrm{kpc}$ ) is potentially less steep in the observations when compared to the simulation (yet remain consistent to within the errors). Focusing now on the excess in the lower panel, we find very similar behaviours for both cuts of AGN luminosity from the simulation and also from the galaxies within the observed sample. At larger separations ( $r \gtrsim 40$ projected $\mathrm{kpc}$ ) there is no notable excess in the AGN fraction relative to their isolated control galaxies, but at smaller separations $(r \lesssim 40$ projected kpc) a trend of a rising excess with decreasing pair separation begins to appear, reaching excess values of around $\approx 1.5$ (albeit with large errors).

Therefore the results from the simulation and the observations are encouragingly alike, both showing a quantitatively similar degree of evidence for an increased amount of AGN activity for galaxies with close major companions, consistent with the overall results of this study.

\subsection{Is the enhancement of $\mathrm{BH}$ activity during major mergers important for $\mathrm{BH}$ growth?}

In this study we have investigated the relationship between galaxygalaxy mergers and enhanced $\mathrm{BH}$ activity within a cosmological context. We have found that there exists a measurable excess in the fraction of highly accreting BHs that reside in major mergers relative to those that reside in isolated systems, through both the merger fraction of AGN and the AGN fraction of merging systems. However, it remains difficult to gauge from the values of the fractional 'excess' alone how important major mergers are for producing luminous or high Eddington rate AGN, and if the enhanced $\mathrm{BH}$ growth resulting from this process is statistically meaningful. Or, more fundamentally, would the $\mathrm{BH}$ population today look the same in a Universe free from any major interactions (which can now be investigated for the evolution of individual systems, e.g. Pontzen et al. 2017, but not for global populations). We note that when we refer to an enhancement of $\mathrm{BH}$ growth, here we are referring to the increased accretion on to BHs directly triggered by the merger process, and not the growth resulting from the coalescence of two BHs.

Panel A of Fig. 10 shows the cosmic black hole accretion rate density (BHARD) from all galaxies more massive than $M_{*} \geq$ $10^{10} \mathrm{M}_{\odot}$ in the EAGLE simulation, showing also the contribution from the subset of these galaxies currently in the state of a major merger (i.e. $\left|n_{\text {dyn[Major }]}\right| \leq 1$ ) and those not currently in the state of a major merger (i.e. $\left.\left|n_{\text {dyn[Major] }}\right|>1\right)$. At the highest redshifts $(z \geq$ 3 ), the galaxies currently experiencing a major merger contribute the greatest amount to the total BHARD ( $\gtrsim 70$ per cent, panel B). However we note that at these redshifts the majority of galaxies above $M_{*} \geq 10^{10} \mathrm{M}_{\odot}$ are in a merging state (panel C). During intermediate redshifts $(z \approx 2)$ both merging and isolated systems contribute a similar amount to the total BHARD, even although the majority of systems by this time are not experiencing a major merger. As we evolve towards the present day $(z=0)$, isolated systems have come to dominate both the galaxy population by number $(\approx 97$ percent, panel $\mathrm{C})$ and the contribution to the total BHARD $(\approx 90$ per cent, panel B).

The galaxies currently experiencing a major merger always contribute more to the total BHARD relative to their abundance, i.e. the ratio between $f_{\mathrm{BHARD}}$ and $f_{N}$ is always $>1$ (panel D). In other words, the average accretion rate of merging galaxies is always higher than the average accretion rate of all galaxies (i.e. $\left.\left\langle\dot{m}_{\mathrm{BH}[\text { Mergers }]}\right\rangle /\left\langle\dot{m}_{\mathrm{BH}[\mathrm{All}]}\right\rangle>1\right)$, growing from a factor of $\approx 1.2$ at higher redshifts $(z>1)$ up to a factor of $\approx 3$ at $z=0$ (panel D). Relative to the accretion rate of isolated galaxies however, merging galaxies are always accreting on average at a 2-3 times higher rate 


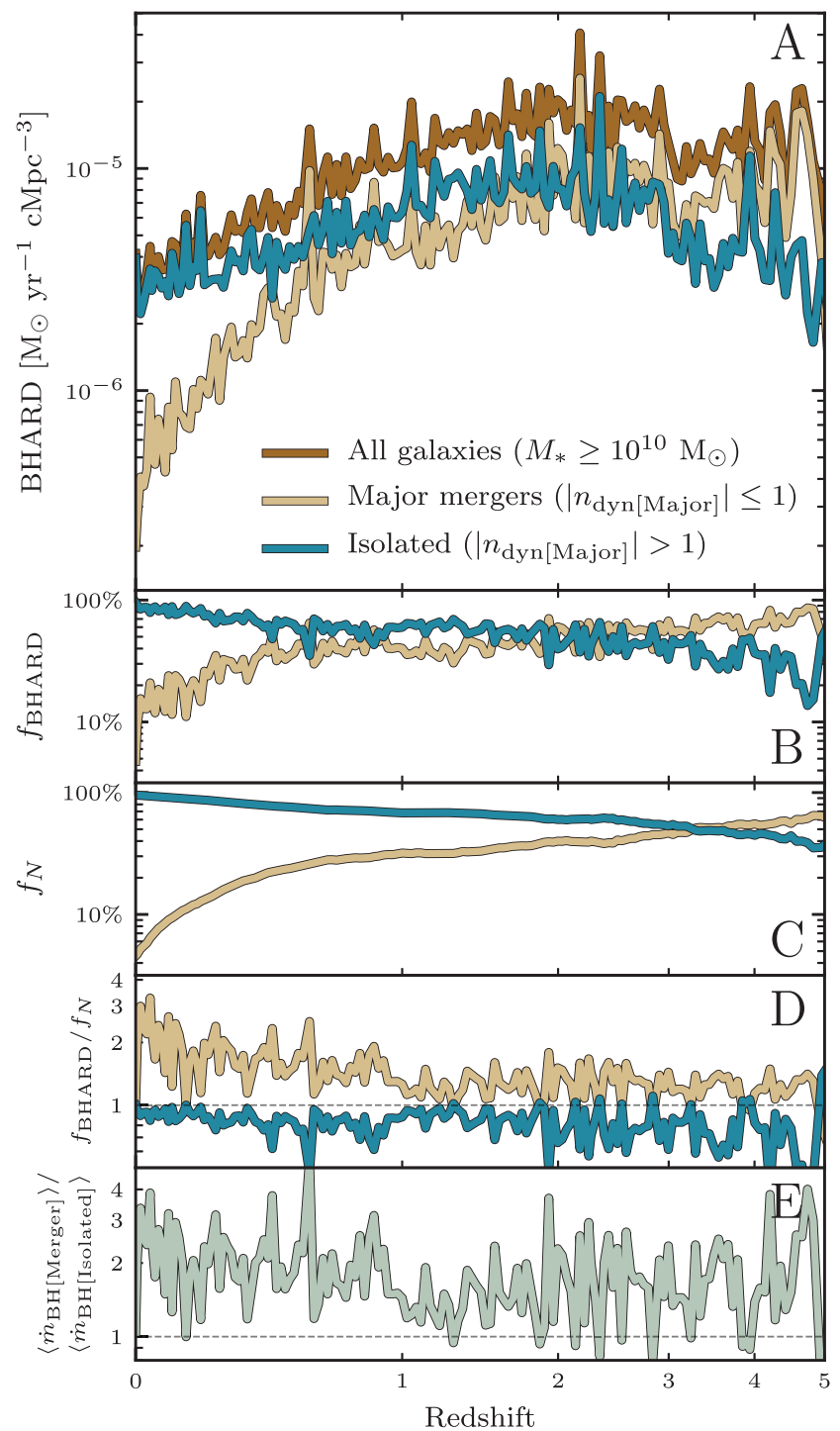

Figure 10. Panel A: the $\mathrm{BH}$ accretion rate density (BHARD) from all galaxies more massive than $M_{*} \geq 10^{10} \mathrm{M}_{\odot}$, galaxies currently in the state of a major merger (i.e. $\left|n_{\text {dyn[Major] }}\right| \leq 1$ ) and galaxies not currently in the state of a major merger (i.e. $\left|n_{\mathrm{dyn}[\mathrm{Major}]}\right|>1$ ). Panel B: the fraction of the total BHARD coming from merging and non-merging systems. Panel C: the fraction of these galaxies that are in a major merger and not in a major merger. Panel D: the ratio between panels B and C, i.e. the contribution to the BHARD from merging and non-merging systems weighted by their number, or equivalently, the mean accretion rate of merging and isolated galaxies versus the mean accretion rate of all galaxies. Panel E: the ratio between the mean accretion rate of merging galaxies and the mean accretion rate of isolated galaxies. At higher redshifts $(z \gtrsim 2)$ the majority of the BHARD come from merging galaxies, however the majority of galaxies at this time are in mergers. At lower redshifts $(z \lesssim 2)$ both the galaxy population and the BHARD are dominated by isolated systems. At all redshifts, merging galaxies have accretion rates $\approx 2$ times greater than isolated galaxies.

(panel E). This could suggest that a significantly increased amount of $\mathrm{BH}$ growth can be attributed to the triggering influence of major mergers, particularly at higher redshifts $(z \gtrsim 2)$ where merging systems are the most abundant.

From Fig. 10 we discovered that merging galaxies at all redshifts accrete at an average rate that is $2-3$ times higher than that of isolated galaxies. However, to establish the cumulative impact of

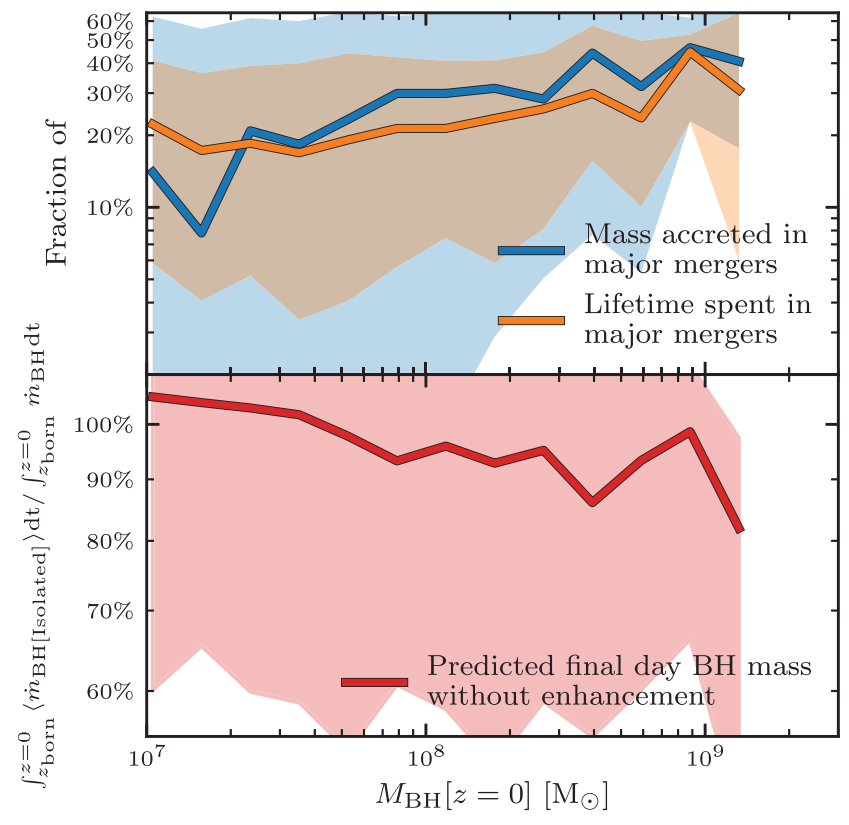

Figure 11. Upper panel: the fraction of the total accreted mass (i.e. $\int_{z \text { born }}^{z=0} \dot{m}_{\mathrm{BH}} \mathrm{dt}$ ) that was accreted during the period(s) of a major merger (i.e. the fraction of mass that was accreted within \pm 1 dynamical time of the coalescence of the two galaxies) as a function of the present day BH mass. To compare, the fraction of the BHs lifetime that was spent in a major merging system is also shown. On average, BHs accumulate an increasing amount of their accreted mass with increasing present day $\mathrm{BH}$ mass during the period(s) of a major merger $\left(\approx 40\right.$ per cent at $\left.M_{\mathrm{BH}[\mathrm{z}=0]}=10^{9} \mathrm{M}_{\odot}\right)$, but also spend an increasing fraction of their lifetimes within a merging system with increasing present day BH mass. During the period(s) of a major merger, the average $\mathrm{BH}$ never accumulates more than 50 per cent of their accreted mass. Lower panel: the predicted final day $\mathrm{BH}$ mass if $\mathrm{BHs}$ did not experience any enhancement in their growth triggered via a major merger, relative to the true final day BH mass. The predicted mass is obtained by multiplying the average $\mathrm{BH}$ accretion rate during times of isolation by the total lifetime of the BH. On average, we predict BHs would still be $\gtrsim 85$ per cent of their true mass if mergers did not enhance $\mathrm{BH}$ activity.

this enhancement upon the resulting $\mathrm{BH}$ growth we must look at the $\mathrm{BH}$ accretion rate histories of galaxies. In Fig. 11 we show the fraction of the total accreted mass (i.e. $\int_{z_{\text {born }}}^{z=0} \dot{m}_{\mathrm{BH}} \mathrm{dt}$ ) that was accreted during the period(s) of a major merger (i.e. the fraction of mass that was accreted within \pm 1 dynamical time of the coalescence of the two galaxies) as a function of the present day BH mass. Although there is an extremely large scatter, the average $\mathrm{BH}$ with a present day mass of $M_{\mathrm{BH}}=10^{7} \mathrm{M}_{\odot}$ accumulated $\approx 10$ per cent of their accreted mass during the period of a major merger, and this number rises to $\approx 40$ per cent for BHs with a present day mass of $M_{\mathrm{BH}}=10^{9} \mathrm{M}_{\odot}$. To put this in perspective, we additionally show what fraction of the BHs lifetime was spent in a 'merging state', revealing a similar rising trend, but a slightly lower normalization to the mass fractions $(\approx 5-10$ per cent). This reveals yet more evidence that $\mathrm{BHs}$ are accreting proportionally more during their time within a major merger over when they are isolated.

Exploring the $\mathrm{BH}$ accretion rate histories further, we can crudely attempt to estimate what mass a BH would have been if it had never experienced the enhanced accretion rates during a major merger. To do this we evaluate a new present day $\mathrm{BH}$ mass by simply multiplying the average accretion rate a $\mathrm{BH}$ has over its lifetime during isolation (i.e. at all times it is not in a major merger) by the total lifetime of the $\mathrm{BH}$. We then compare this 'non-enhanced' $\mathrm{BH}$ 
mass $\left(\int_{z_{\text {born }}}^{z=0}\left\langle\dot{m}_{\mathrm{BH}[\text { Isolated] }}\right\rangle \mathrm{dt}\right)$ to the true $\mathrm{BH}$ mass $\left(\int_{z_{\text {born }}}^{z=0} \dot{m}_{\mathrm{BH}} \mathrm{dt}\right)$ in the lower panel of Fig. 11. We find that, whilst the scatter is again large, if a $\mathrm{BH}$ was to grow at their mean isolated accretion rate it would typically result in a $\mathrm{BH}$ that grows to over $\gtrsim 85$ percent of the true mass. Or, said in reverse, on average the cumulative result of the enhanced accretion rates triggered via major mergers are responsible for no more than 15 per cent of the final $\mathrm{BH}$ masses at $z=0$ (strongly in line with the conclusions reported by Martin et al. 2018, who also find the majority of $\mathrm{BH}$ growth from the cosmological hydrodynamical HORIZON-AGN simulation occurs outwith mergers).

Thus it remains difficult to definitively state the 'importance' of major mergers for enhancing $\mathrm{BH}$ growth, yet we would argue that overall they are not statistically relevant fuelling mechanisms for BHs. Major mergers do increase the average accretion rates of BHs at all redshifts, by a factor of 2-3 over their isolated counterparts. However this enhancement is either not great enough, or BHs simply do not experience enough cumulative time in a merging state to feel this enhancement in their final BH mass, with the majority of accreted $\mathrm{BH}$ mass being accumulated in an isolated state. It is plausible that mergers do become increasingly important for triggering $\mathrm{BH}$ activity with decreasing redshift, as we have seen multiple times throughout this study. However, by these times merging systems are now so rare that their (albeit enhanced) contribution is still not highly significant, and isolated galaxies remain the dominant source of $\mathrm{BH}$ accretion at lower redshifts. The conclusion of mergers never being statistically relevant fuelling mechanisms for BHs is consistent with the results from the MAGNETICUM PATHFINDER simulation, who performed a similar analysis to this study in the high-mass regime $\left(M_{*} \geq 10^{11} \mathrm{M}_{\odot}\right.$; Steinborn et al. 2018). However we emphasize that if we were to restrict our study to just the high-mass regime $\left(M_{*} \geq\right.$ $10^{11} \mathrm{M}_{\odot}$ ) as they did, we would not have found the same result (see Fig. 4).

Even if major mergers are not important for $\mathrm{BH}$ growth as a whole, they could still remain important drivers for rare, or unique, events. For example, it is plausible that extremely luminous quasars $\left(L_{\mathrm{bol}} \gtrsim 10^{46} \mathrm{erg} \mathrm{s}^{-1}\right)$ cannot be sustained via secular processes, and could therefore require a triggering interaction to occur (we have seen evidence in this study that the most luminous AGN are those most commonly found in merging systems, e.g. Figs 1 and A2). In addition, in McAlpine et al. (2017) we found that the initiation of the 'rapid growth phase' of BHs was commonly found to occur in close proximity to a merger, and, using a sample of control galaxies, found that the importance of mergers for triggering the rapid growth phase increased with decreasing redshift. This directly agrees with the results presented by this study. The BHs experiencing their rapid growth phase are essentially unhindered in their growth, and as such grow close to the Eddington limit. Therefore it is plausible that the strongest signal in the merger fraction excess using the Eddington rates seen in Figs 1, 2, and 8 is largely from the BHs currently experiencing their rapid growth phase.

\subsubsection{Are minor mergers important?}

This study has focused exclusively on the influence of major mergers (i.e. $M_{*, 1} / M_{*, 2} \geq \frac{1}{4}$ ) as triggering mechanisms for increased $\mathrm{BH}$ activity. However, minor mergers may also play a role, and, as they are more common than major mergers, their importance could potentially be much larger. Here we define minor mergers as those with stellar mass ratios of $\frac{1}{10}<M_{*, 1} / M_{*, 2}<\frac{1}{4}$.

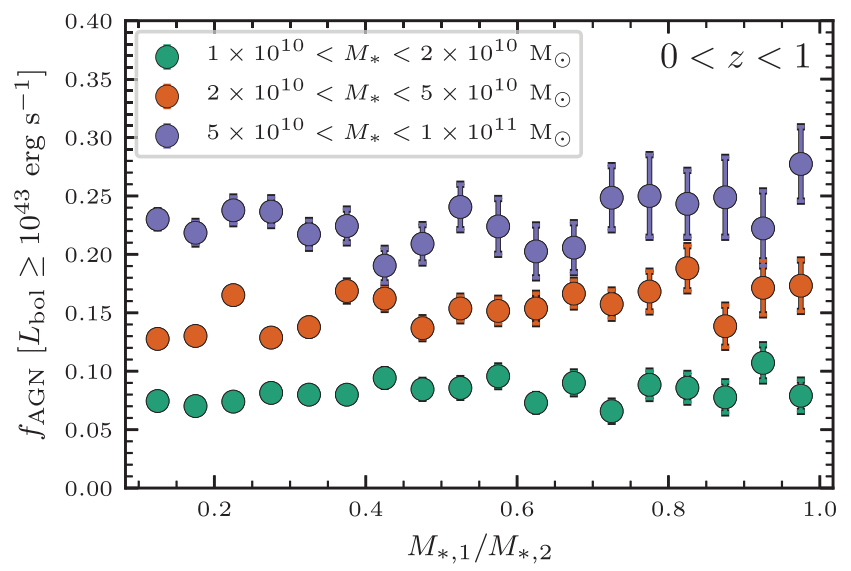

Figure 12. The AGN fraction (i.e. the fraction of galaxies hosting a $\mathrm{BH}$ with a bolometric luminosity $L_{\mathrm{bol}} \geq 10^{43} \mathrm{erg} \mathrm{s}^{-1}$ ) of merging galaxies in the redshift range $0<z<1$ as a function of the stellar mass ratio $\left(M_{*, 1} / M_{*, 2}\right.$, where $M_{*, 2}$ is always the most massive member of the galaxy pair). The galaxies are split into three stellar mass ranges as indicated by the legend, and the error bars indicate the Poisson uncertainty. We find that the AGN fraction of merging galaxies is insensitive to the stellar mass ratio over the range $\frac{1}{10}<M_{*, 1} / M_{*, 2}<1$, which is why we find similar results for this study when considering either minor mergers $\left(\frac{1}{10}<M_{*, 1} / M_{*, 2}<\frac{1}{4}\right)$ or major mergers $\left(M_{*, 1} / M_{*, 2} \geq \frac{1}{4}\right)$.

If we repeat the analysis of this study now for minor mergers we find a very similar overall result: the enhancements of the merger fractions in Fig. 1, the AGN fractions in Figs 2 and 3, and the contribution to the CBHAR in Fig. 10 are all virtually unchanged. We can see why this is from Fig. 12, which shows the AGN fraction of merging galaxies in the redshift range $0<z<1$ as a function of the stellar mass ratio $\left(M_{*, 1} / M_{*, 2}\right)$. Whilst one may have expected an increasing influence upon $\mathrm{BH}$ activity with increasing stellar mass ratio, instead, over the stellar mass ratio range $\frac{1}{10}<M_{*, 1} / M_{*, 2}<1$ the AGN fraction of galaxies does not evolve.

Therefore the conclusions we have reported for major mergers also hold true for minor mergers, in that they do enhance BH activity, yet this enhancement is not statistically meaningful for $\mathrm{BH}$ growth.

\section{CONCLUSIONS}

Using the cosmological hydrodynamical EAGLE simulation, we have investigated to what degree black hole $(\mathrm{BH})$ activity is enhanced during the period of a major merger (i.e. those with a stellar mass ratio of $M_{*, 1} / M_{*, 2} \geq \frac{1}{4}$, where $M_{*, 2}$ is the most massive of the two galaxies). For this study, an 'active' BH (or AGN) is defined to be one that has a bolometric AGN luminosity greater than $L_{\text {bol }} \geq 10^{43} \mathrm{erg} \mathrm{s}^{-1}$ or an Eddington rate greater than $\lambda_{\text {edd }} \geq 10^{-2}$ ('inactive' galaxies are therefore those with BHs accreting at rates lower than these limits, i.e. $L_{\text {bol }}<10^{43} \mathrm{erg} \mathrm{s}^{-1}$ or $\lambda_{\text {edd }}<10^{-2}$ ). When referring to an 'excess' value below, we are referring to the ratio of two merger or AGN fractions (between the merger or AGN fractions of the selected samples and their associated control samples).

Our main conclusions are as follows:

(i) AGNs have a higher major merger fraction than their inactive galaxy counterparts. The excess between the major merger fraction of AGN relative to the major merger fraction of inactive galaxies increases with increasing AGN luminosity and Eddington rate: reaching a factor of $\approx 1.75$ at $L_{\text {bol }} \sim 10^{4} \mathrm{erg} \mathrm{s}^{-1}$, and a factor of $\approx 3$ at the Eddington limit (see Fig. 1). 
(ii) Their AGN fraction of major mergers is higher than the AGN fraction of their isolated galaxy counterparts. The excess between the AGN fraction of merging and isolated systems, defined by either a cut in the AGN luminosity or Eddington rate, increases with decreasing stellar mass at $z<1$ (up to a maximum value of $\approx 1.8$ at $\left.M_{*} \approx 10^{10} \mathrm{M}_{\odot}\right)$. At higher redshifts $(z>1)$, the excess in the AGN fraction remains approximately constant for all stellar masses (with a value of $\approx 1.1-1.4$, see Fig. 2 ).

(iii) The AGN fraction of galaxies with close major companions is higher than the AGN fraction of their isolated galaxy counterparts. When an AGN is defined by a cut in the bolometric luminosity, there is a potential slight excess between the AGN fraction of galaxies with close major companions and isolated systems, oscillating around a value of $\approx 1.1$ for $3 \mathrm{D}$ separations lower than $r_{\text {sep[Major] }} \lesssim 80$ pkpc. However, when an AGN is defined by a cut in the Eddington rate, a strong trend of an increasing excess with decreasing 3D separation is found for galaxies at $z<2$, starting at $3 \mathrm{D}$ separations of $50 \leq r_{\text {sep[Major] }} \leq 100 \mathrm{pkpc}$, and rising to an excess value of $1.2-1.3$ at $3 \mathrm{D}$ separations of $\approx 10 \mathrm{pkpc}$ (see Figs 3 and 9).

(iv) The galaxies hosting the BHs with the greatest enhancement of BH activity due to a major merger are almost exclusively lower mass $\left(\boldsymbol{M}_{*} \lesssim \mathbf{1 0}^{11} \mathbf{M}_{\odot}\right)$. We find little to no enhancement of BH activity in massive $\left(M_{*} \gtrsim 10^{11} \mathrm{M}_{\odot}\right)$ active or merging systems relative to their inactive or isolated counterparts. In addition, the galaxies with the largest excess in their merger and AGN fractions above the control samples are those with higher gas fractions $\left(f_{\text {gas }} \gtrsim\right.$ $0.2)$, less massive BHs $\left(M_{\mathrm{BH}} \lesssim 10^{7} \mathrm{M}_{\odot}\right)$ and those that are central galaxies (see Figs 2 and 4 to 6 ).

(v) The majority of $\mathrm{BH}$ activity triggered via a major merger resides within the early remnants of merging systems. At higher redshifts $(z>1), \approx 50$ percent of the $\mathrm{BH}$ activity triggered via a major merger occurs during the dynamical time after the two galaxies have already coalesced. At lower redshifts $(z<1)$, this fraction raises to $\approx 65-75$ per cent. In addition, at lower redshifts $(z$ $<1$ ) the peak of triggered $\mathrm{BH}$ activity occurs $\approx 0.25$ dynamical times $(\approx 300 \mathrm{Myr}$ at $z=0.5)$ after the coalescence of the two galaxies, suggesting that there is typically a significant delay between the coalescence of the two galaxies and triggered BH activity (see Figs 7 and 8).

(vi) The excess values of both the merger fraction of AGN and the AGN fraction of merging systems increases with decreasing redshift. Throughout our analysis we have consistently found higher excess values between the merger fraction of AGN and inactive galaxies and between the AGN fraction of merging systems and isolated galaxies with decreasing redshift. This suggests that mergers are becoming increasingly important for triggering $\mathrm{BH}$ activity as the universe evolves (see Figs 1 to 3, 7, and 8). However, the abundance of merging systems does substantially decrease with decreasing redshift (see Fig. 10).

(vii) Overall, mergers are not statistically relevant fuelling mechanisms for BHs. Whilst we have repeatably found that mergers are enhancing the amount of AGN activity within the EAGLE simulation, we would argue that major (or minor see Section 4.3.1) mergers, as triggering mechanisms, do not contribute a significant amount to $\mathrm{BH}$ growth globally. Both at higher redshifts $(z \gtrsim 2)$, when the majority of galaxies more massive than $M_{*} \geq 10^{10} \mathrm{M}_{\odot}$ are currently experiencing a merger, and at lower redshifts $(z \lesssim 2)$, when major mergers have become a small minority of the galaxy population, merging systems typically have accretion rates that are on average 2-3 times greater than their isolated counterparts (see Fig. 10). However, either this level of enhancement is too small, or the time spent within major mergers is too short, to have a meaningful impact upon the final day $\mathrm{BH}$ mass. The BHs at the present day have, on average, accumulated the majority of their mass outwith the period(s) of a major merger (see Fig. 11). Indeed, we estimate that $\mathrm{BHs}$ in a universe where major interactions did not enhance $\mathrm{BH}$ activity would have $\mathrm{BH}$ masses that were $\gtrsim$ 85 percent of the mass of BHs in the true EAGLE universe (see Fig. 11). Therefore it appears that the enhanced growth triggered via a merger is not a necessary component for global BH growth, and the $\mathrm{BH}$ population would potentially be very similar in a Universe that was absent of this enhancement. However this does not rule out the importance, or necessity, for mergers to trigger unique events in a BHs lifetime, such as for the creation of the most highly luminous quasars, or for initiating the rapid growth phase of BHs (McAlpine et al. 2018).

\section{ACKNOWLEDGEMENTS}

We thank the referee for their comments that have improved the quality of this work. This work was supported by the Academy of Finland (grant number 314238). PHJ acknowledges the support by the European Research Council via ERC Consolidator Grant KETJU (no. 818930). DRP and SLE gratefully acknowledge NSERC for Discovery Grants which helped to fund this research.

This work used the DiRAC@ Durham facility managed by the Institute for Computational Cosmology on behalf of the STFC DiRAC HPC Facility (www.dirac.ac.uk). The equipment was funded by BEIS capital funding via STFC capital grants ST/K00042X/1, ST/P002293/1, ST/R002371/1 and ST/S002502/1, Durham University and STFC operations grant ST/R000832/1. DiRAC is part of the National e-Infrastructure.

Funding for the SDSS and SDSS-II has been provided by the Alfred P. Sloan Foundation, the Participating Institutions, the National Science Foundation, the U.S. Department of Energy, the National Aeronautics and Space Administration, the Japanese Monbukagakusho, the Max Planck Society, and the Higher Education Funding Council for England. The SDSS Web Site is http://www.sdss.org/.

The SDSS is managed by the Astrophysical Research Consortium for the Participating Institutions. The Participating Institutions are the American Museum of Natural History, Astrophysical Institute Potsdam, University of Basel, University of Cambridge, Case Western Reserve University, University of Chicago, Drexel University, Fermilab, the Institute for Advanced Study, the Japan Participation Group, Johns Hopkins University, the Joint Institute for Nuclear Astrophysics, the Kavli Institute for Particle Astrophysics and Cosmology, the Korean Scientist Group, the Chinese Academy of Sciences (LAMOST), Los Alamos National Laboratory, the MaxPlanck-Institute for Astronomy (MPIA), the Max-Planck-Institute for Astrophysics (MPA), New Mexico State University, Ohio State University, University of Pittsburgh, University of Portsmouth, Princeton University, the United States Naval Observatory, and the University of Washington.

\section{REFERENCES}

Barnes J. E., Hernquist L. E., 1991, ApJ, 370, L65

Blumenthal K. A., Barnes J. E., 2018, MNRAS, 479, 3952

Bondi H., Hoyle F., 1944, MNRAS, 104, 273

Bottrell C. et al., 2019, MNRAS, 490, 5390

Brandt W. N., Alexander D. M., 2015, A\&AR, 23, 1

Chabrier G., 2003, PASP, 115, 763 
Cisternas M. et al., 2011, ApJ, 726, 57

Cotini S., Ripamonti E., Caccianiga A., Colpi M., Della Ceca R., Mapelli M., Severgnini P., Segreto A., 2013, MNRAS, 431, 2661

Crain R. A. et al., 2015, MNRAS, 450, 1937

Dalla Vecchia C., Schaye J., 2012, MNRAS, 426, 140

Di Matteo T., Springel V., Hernquist L., 2005, Nature, 433, 604

Dolag K., Borgani S., Murante G., Springel V., 2009, MNRAS, 399, 497

Dubois Y., Volonteri M., Silk J., Devriendt J., Slyz A., Teyssier R., 2015, MNRAS, 452, 1502

Ellison S. L., Patton D. R., Simard L., McConnachie A. W., Baldry I. K., Mendel J. T., 2010, MNRAS, 407, 1514

Ellison S. L., Patton D. R., Mendel J. T., Scudder J. M., 2011, MNRAS, 418, 2043

Ellison S. L., Mendel J. T., Patton D. R., Scudder J. M., 2013, MNRAS, 435,3627

Ellison S. L., Patton D. R., Hickox R. C., 2015, MNRAS, 451, L35

Ellison S. L., Viswanathan A., Patton D. R., Bottrell C., McConnachie A. W., Gwyn S., Cuillandre J.-C., 2019, MNRAS, 487, 2491

Fan L. et al., 2016, ApJ, 822, L32

Furlong M. et al., 2015, MNRAS, 450, 4486

Furlong M. et al., 2017, MNRAS, 465, 722

Glikman E., Simmons B., Mailly M., Schawinski K., Urry C. M., Lacy M., 2015, ApJ, 806, 218

Goulding A. D., Alexander D. M., 2009, MNRAS, 398, 1165

Goulding A. D. et al., 2018, PASJ, 70, S37

Hewlett T., Villforth C., Wild V., Mendez-Abreu J., Pawlik M., Rowlands K., 2017, MNRAS, 470, 755

Hickox R. C., Mullaney J. R., Alexander D. M., Chen C.-T. J., Civano F. M., Goulding A. D., Hainline K. N., 2014, ApJ, 782, 9

Hirschmann M., Khochfar S., Burkert A., Naab T., Genel S., Somerville R. S., 2010, MNRAS, 407, 1016

Hirschmann M., Dolag K., Saro A., Bachmann L., Borgani S., Burkert A., 2014, MNRAS, 442, 2304

Hopkins P. F., Hernquist L., Cox T. J., Kereš D., 2008, ApJS, 175, 356

Jahnke K., Macciò A. V., 2011, ApJ, 734, 92

Ji I., Peirani S., Yi S. K., 2014, A\&A, 566, A97

Johansson P. H., Burkert A., Naab T., 2009, ApJ, 707, L184

Kauffmann G. et al., 2003, MNRAS, 341, 33

Kocevski D. D. et al., 2012, ApJ, 744, 148

Koss M., Mushotzky R., Veilleux S., Winter L., 2010, ApJ, 716, L125

Koss M. J. et al., 2018, Nature, 563, 214

Lahén N., Johansson P. H., Rantala A., Naab T., Frigo M., 2018, MNRAS, 475,3934

Lotz J. M., Jonsson P., Cox T. J., Primack J. R., 2010, MNRAS, 404, 575

Magorrian J. et al., 1998, AJ, 115, 2285

Marian V. et al., 2019, ApJ, 882, 141

Martin G. et al., 2018, MNRAS, 476, 2801

McAlpine S. et al., 2016, Astron. Comput., 15, 72

McAlpine S., Bower R. G., Harrison C. M., Crain R. A., Schaller M., Schaye J., Theuns T., 2017, MNRAS, 468, 3395

McAlpine S., Bower R. G., Rosario D. J., Crain R. A., Schaye J., Theuns T., 2018, MNRAS, 481, 3118

McConnell N. J., Ma C.-P., 2013, ApJ, 764, 184

Mechtley M. et al., 2016, ApJ, 830, 156

Mihos J. C., Hernquist L., 1996, ApJ, 464, 641

Patton D. R., Torrey P., Ellison S. L., Mendel J. T., Scudder J. M., 2013, MNRAS, 433, L59

Patton D. R., Qamar F. D., Ellison S. L., Bluck A. F. L., Simard L., Mendel J. T., Moreno J., Torrey P., 2016, MNRAS, 461, 2589

Pawlik M. M., Wild V., Walcher C. J., Johansson P. H., Villforth C., Rowlands K., Mendez-Abreu J., Hewlett T., 2016, MNRAS, 456, 3032 Peng C. Y., 2007, ApJ, 671, 1098

Planck Collaboration et al., 2014, A\&A, 571, A1

Pontzen A., Tremmel M., Roth N., Peiris H. V., Saintonge A., Volonteri M., Quinn T., Governato F., 2017, MNRAS, 465, 547

Qu Y. et al., 2017, MNRAS, 464, 1659

Rantala A., Pihajoki P., Johansson P. H., Naab T., Lahén N., Sawala T., 2017, ApJ, 840, 53
Rodighiero G. et al., 2015, ApJ, 800, L10

Rodríguez Montero F., Davé R., Wild V., Anglés-Alcázar D., Narayanan D., 2019, MNRAS, 490, 2139

Rodriguez-Gomez V. et al., 2015, MNRAS, 449, 49

Rosario D. J. et al., 2015, A\&A, 573, A85

Rosas-Guevara Y. M. et al., 2015, MNRAS, 454, 1038

Rosas-Guevara Y., Bower R. G., Schaye J., McAlpine S., Dalla Vecchia C., Frenk C. S., Schaller M., Theuns T., 2016, MNRAS, 462, 190

Salcido J., Bower R. G., Theuns T., McAlpine S., Schaller M., Crain R. A., Schaye J., Regan J., 2016, A\&A, 463, 870

Sanders D. B., Soifer B. T., Elias J. H., Madore B. F., Matthews K., Neugebauer G., Scoville N. Z., 1988, ApJ, 325, 74

Satyapal S., Ellison S. L., McAlpine W., Hickox R. C., Patton D. R., Mendel J. T., 2014, MNRAS, 441, 1297

Schawinski K., Treister E., Urry C. M., Cardamone C. N., Simmons B., Yi S. K., 2011, ApJ, 727, L31

Schawinski K., Simmons B. D., Urry C. M., Treister E., Glikman E., 2012, MNRAS, 425, L61

Schawinski K., Koss M., Berney S., Sartori L. F., 2015, MNRAS, 451, 2517

Schaye J., Dalla Vecchia C., 2008, MNRAS, 383, 1210

Schaye J. et al., 2015, MNRAS, 446, 521

Scholtz J. et al., 2018, MNRAS, 475, 1288

Shakura N. I., Sunyaev R. A., 1973, A\&A, 24, 337

Silverman J. D. et al., 2011, ApJ, 743, 2

Snyder G. F., Rodriguez-Gomez V., Lotz J. M., Torrey P., Quirk A. C. N., Hernquist L., Vogelsberger M., Freeman P. E., 2019, MNRAS, 486, 3702

Springel V., White S. D. M., Tormen G., Kauffmann G., 2001, MNRAS, 328,726

Springel V., Di Matteo T., Hernquist L., 2005a, ApJ, 620, L79

Springel V., Di Matteo T., Hernquist L., 2005b, MNRAS, 361, 776

Steinborn L. K., Hirschmann M., Dolag K., Shankar F., Juneau S., Krumpe M., Remus R.-S., Teklu A. F., 2018, MNRAS, 481, 341

The EAGLE team, 2017, preprint (arXiv:1706.09899)

Trayford J. W. et al., 2015, MNRAS, 452, 2879

Villforth C. et al., 2014, MNRAS, 439, 3342

Villforth C. et al., 2017, MNRAS, 466, 812

Volonteri M., Capelo P. R., Netzer H., Bellovary J., Dotti M., Governato F., 2015, MNRAS, 449, 1470

Weston M. E., McIntosh D. H., Brodwin M., Mann J., Cooper A., McConnell A., Nielsen J. L., 2017, MNRAS, 464, 3882

Wiersma R. P. C., Schaye J., Smith B. D., 2009a, MNRAS, 393, 99

Wiersma R. P. C., Schaye J., Theuns T., Dalla Vecchia C., Tornatore L., 2009b, MNRAS, 399, 574

Zolotov A. et al., 2015, MNRAS, 450, 2327

\section{APPENDIX A: CHOICE OF PARAMETERS}

In this appendix we explore how sensitive the results of this study are to our choice of parameters, that is: the definition of a 'merging state', the definition of an 'active' BH and how we match a selected galaxy to a control galaxy. We note, that in Appendix A1 we only explicitly describe the changes to the results when considering the bolometric AGN luminosity, as the differences when considering the Eddington rate are so similar. In Appendix A2 we explicitly describe the changes when considering both the bolometric AGN luminosity and Eddington rate separately.

\section{A1 Defining a 'merging state' and an 'active' BH}

In Section 3.1.1 we investigated the merger fraction of AGN by assuming that galaxies were in the 'state of a merger' if they have recently undergone coalescence with another galaxy up to one dynamical time in the past, or will undergo coalescence with another galaxy up to one dynamical time in the future (i.e. $a=-1$ and $b=1$ in equation 5). This allowed us to identity major merging 


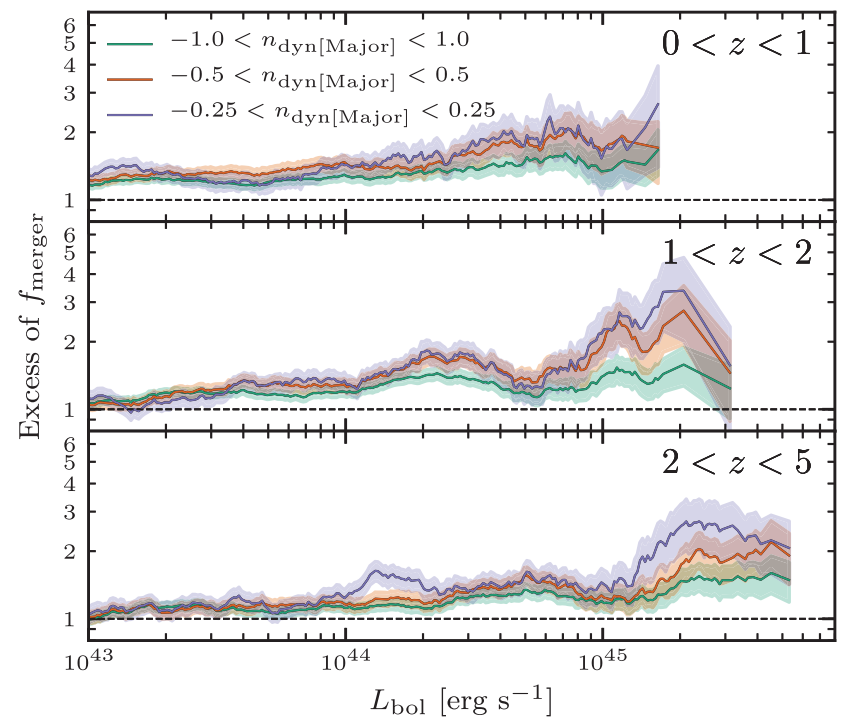

Figure A1. How the excess in the major merger fraction of AGN as a function of the bolometric AGN luminosity (i.e. the results from lower lefthand panel of Fig. 1) varies with how we define a 'merging state' (see equation 5). Our fiducial value, $-1.0<n_{\mathrm{dyn}[\text { Major }]}<1.0$, typically produces lower excess values than if we were to consider a smaller dynamical time window, with the excess values potentially varying by up to a factor of $\approx 2$ at the highest AGN luminosities, i.e. $L_{\mathrm{bol}} \gtrsim 10^{45} \mathrm{erg} \mathrm{s}^{-1}$, depending on the choice of dynamical time window (albeit with large errors).

systems as those with a value of $\left|n_{\mathrm{dyn}[\text { Major] }}\right| \leq 1$, and 'isolated' systems as those with a value of $\left|n_{\text {dyn[Major] }}\right|>1$ (although for this study we used $\left|n_{\text {dyn[Major] }}\right|>2$ to classify an isolated system to be conservative). Whilst one dynamical time has physical meaning in relation to the dynamics of a system during an interaction, it is still somewhat an arbitrary choice.

In Fig. A1 we investigate how the excess of the merger fraction between galaxies with active and inactive $\mathrm{BHs}$ (i.e. the results from the lower left-hand panel of Fig. 1) varies as we vary the definition of a merging state. We compare the results of our fiducial definition of \pm 1 dynamical time to two shorter dynamical time windows: \pm 0.5 dynamical times and \pm 0.25 dynamical times. Typically, the excess values are largest when considering a smaller dynamical time window, potentially by up to a factor of $\approx 2$ at brighter AGN luminosities (i.e. $\left.L_{\mathrm{bol}} \gtrsim 10^{45} \mathrm{erg} \mathrm{s}^{-1}\right)$ and higher redshifts $(z>$ 1). This results from the fact that the greatest enhancement of $\mathrm{BH}$ activity triggered via the merger process comes around or soon after the coalescence of the two galaxies has completed (i.e. close to $n_{\text {dyn[Major] }} \approx 0$, see Figs 7 and 8 ). We recognize that the regions where the increased excess is largest (i.e. at higher AGN luminosities) is also the region with the largest errors, and thus the values do still remain consistent with one another. Regardless, the overall behaviour of a rising excess with rising AGN luminosity appears to be largely independent of the choice of dynamical time window.

In Sections 3.1.2 and 3.1.3 we compared the AGN fraction of merging $\left(\left|n_{\text {dyn[Major }]}\right| \leq 1\right)$ and isolated galaxies $\left(\left|n_{\text {dyn[Major }]}\right|>2\right)$ as a function of stellar mass (see Fig. 2), and the 3D pair separation (see Fig. 3). This required us to make a choice of cut to define what is and what isn't an 'active' BH. For this study, when defined by a cut in the bolometric AGN luminosity an active $\mathrm{BH}$ has a value greater than $L_{\mathrm{bol}} \geq 10^{43} \mathrm{erg} \mathrm{s}^{-1}$, and when defined by a cut in the Eddington rate an active $\mathrm{BH}$ has a value greater than $\lambda_{\text {edd }} \geq 10^{-2}$.

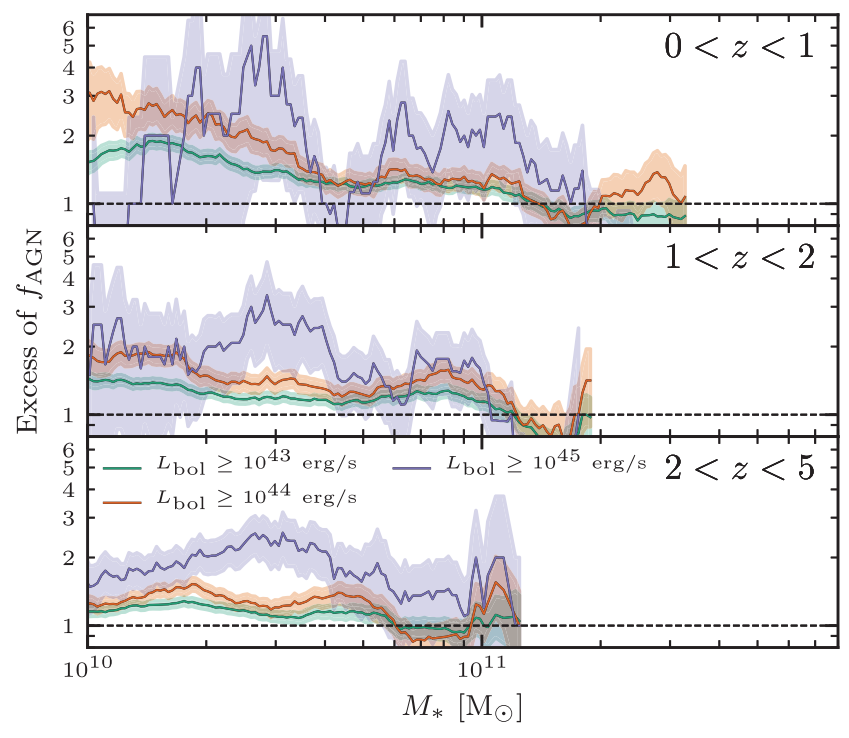

Figure A2. How the excess in the AGN fraction (defined by a cut in the bolometric AGN luminosity) as a function of the stellar mass (i.e. the results from lower left-hand panel of Fig. 2) varies with how we define an 'active' BH. Our fiducial cut, $L_{\mathrm{bol}} \geq 10^{43} \mathrm{erg} \mathrm{s}^{-1}$, typically produces lower excess values than if we were to consider a higher cut in the bolometric luminosity, with the excess values potentially varying by up to a factor of $\approx 3-4$ at lower redshifts depending on the choice of cut (albeit with large errors).

In Fig. A2 we test how the choice of bolometric AGN luminosity cut affects the excess in the AGN fraction between major merging and isolated systems (i.e. the results from the lower left-hand panel of Fig. 2). We compare our fiducial cut of $L_{\text {bol }} \geq 10^{43} \mathrm{erg} \mathrm{s}^{-1}$ to two higher luminosity cuts: $L_{\mathrm{bol}} \geq 10^{44} \mathrm{erg} \mathrm{s}^{-1}$ and $L_{\mathrm{bol}} \geq 10^{45} \mathrm{erg} \mathrm{s}^{-1}$. Typically, higher cuts in the AGN luminosity result in higher excess values: increasing by up to a factor of $\approx 2$ at higher redshifts ( 1 $<z<5$ ) and potentially increasing by up to a factor of $\approx 3$ 4 at lower redshifts $(0<z<1$, albeit with large errors $)$. At redshifts below $z<2$, the trends of an increasing excess in the AGN fraction with decreasing stellar mass are also much more pronounced at the highest AGN luminosity cut we explore $\left(L_{\mathrm{bol}}\right.$ $\geq 10^{45} \mathrm{erg} \mathrm{s}^{-1}$ ), however the overall behaviour is largely similar regardless of the luminosity cut. These results suggest that the excess values are potentially quite sensitive to the choice of AGN cut.

Similarly, in Fig. A3 we test how the choice of bolometric AGN luminosity cut affects the excess in the AGN fraction between galaxies with close major companions and isolated galaxies (i.e. the results from the lower left-hand panel of Fig. 3). We compare our fiducial cut of $L_{\mathrm{bol}} \geq 10^{43} \mathrm{erg} \mathrm{s}^{-1}$ to a higher luminosity cut of $L_{\text {bol }} \geq 10^{44} \mathrm{erg} \mathrm{s}^{-1}$ (luminosity cuts any higher than this have too few numbers to adequately explore within the simulation). Similar to Fig. A2, we find the greatest excess in the AGN fractions above the isolated control galaxies come with higher luminosity cuts (at least for redshifts $z<2$ ).

Therefore the choice of how we define a 'merging state' and 'active' BH does impact the results, and therefore needs to be considered when comparing to similar studies of this nature. It is also for this reason why one should be careful when comparing the merger and AGN fractions, and the resulting excess values, between the predictions of the simulation and the observations. 


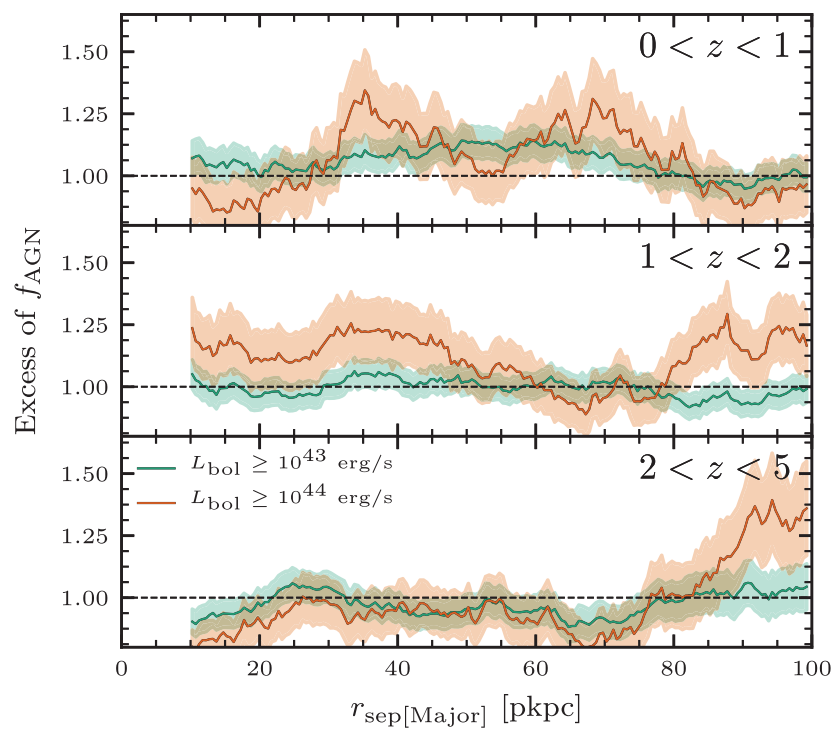

Figure A3. How the excess in the AGN fraction (defined by a cut in the bolometric AGN luminosity) as a function of the 3D pair separation between the two galaxies (i.e. the results from the lower left-hand panel of Fig. 3) varies with how we define an 'active' BH. Our fiducial cut, $L_{\text {bol }}$ $\geq 10^{43} \mathrm{erg} \mathrm{s}^{-1}$, typically produces lower excess values than if we were to consider a higher cut in the bolometric luminosity.

\section{A2 The choice of parameters to match a selected galaxy to a control galaxy for forming a control sample}

Throughout this study we have investigated to what extent galaxygalaxy mergers enhance $\mathrm{BH}$ activity, which we've chosen to quantify by a fractional 'excess' in BH activity relative to a control sample. For Section 3.1.1 it was the excess between the merger fraction of AGN ( $L_{\mathrm{bol}} \geq 10^{43} \mathrm{erg} \mathrm{s}^{-1}$ or $\left.\lambda_{\text {edd }} \geq 10^{-2}\right)$ and a control sample of inactive galaxies $\left(L_{\text {bol }}<10^{43} \mathrm{erg} \mathrm{s}^{-1}\right.$ or $\lambda_{\text {edd }}<10^{-2}$ ), for Section 3.1.2 it was the excess between the AGN fraction of merging systems $\left(\left|n_{\text {dyn[Major }]}\right| \leq 1\right)$ and a control sample of isolated galaxies $\left(\left|n_{\text {dyn[Major] }}\right|>2\right)$, and for Section 3.1.3 it was the excess between the AGN fraction of galaxies with major close companions $\left(r_{\text {sep[Major] }}\right.$ $\leq 100 \mathrm{pkpc})$ and a control sample of isolated galaxies $\left(r_{\text {sep[Major] }}>\right.$ $200 \mathrm{pkpc}$ ). The interpretation of our results, therefore, is sensitive to the value of this excess, which is sensitive to how the galaxies within the selected sample are matched to a control galaxy counterpart (see Section 2.3.1). Here we investigate to what extent the matching criteria by which we choose our control galaxies impacts our results.
It has been well established that: (1) the merger fraction of galaxies at fixed mass increases with increasing redshift, and (2) the merger fraction of galaxies at fixed redshift increases with increasing mass (e.g. Rodighiero et al. 2015; Qu et al. 2017). It is therefore essential that any paired control galaxy must at least match on the stellar mass and redshift. This two-part criteria is how many observational studies of this nature have selected their control galaxies, as it is often all that can be feasibly achieved. Some observational studies have extended this minimalist criteria by also considering the role the environment, by additionally matching the control galaxies on the $N_{2}$ and $r_{2}$ parameters (e.g. Patton et al. 2013, 2016). For this study we wanted to ensure that the control galaxies were as similar as possible to the selected galaxies, opting for a criteria that matches on the stellar, gas, $\mathrm{BH}$, and halo masses and also on the $\mathrm{N}_{2}$ and $r_{2}$ parameters.

To test their impact, here we experiment with three matching criteria:

(i) A 'basic' criteria, matching only on the stellar mass and redshift $\left(M_{*}+z\right)$.

(ii) An 'intermediate' criteria, which additionally matches on the environment $\left(M_{*}+z+N_{2}+r_{2}\right)$.

(iii) A 'strict' criteria, which further matches on the BH mass, gas mass, and halo mass $\left(M_{*}+z+N_{2}+r_{2}+M_{\mathrm{BH}}+M_{\mathrm{gas}}+\right.$ $\left.M_{200}\right)$.

We note that we have deliberately chosen to avoid matching on the SFR, as the SFR of a galaxy can also be enhanced during the merger process.

To see how the choice of matching criteria impacts the results of this study, we include Fig. A4. This shows the excess merger and AGN fractions from the lower panels of Figs 1 to 3, now repeating the analysis for each of the three matching criteria. We find that when the bolometric AGN luminosity is considered, the control pairing criteria has only a slight overall impact on the measured excess. The measured excess is slightly more sensitive to the control pairing criteria when the Eddington rate is considered, however the values never deviate from one another by more than a factor of two, and their errors are often overlapping. Typically, when fewer parameters are matched, the higher the values of the excess. However, regardless of the matching criteria used, the behaviour of the trends is unchanged. Therefore whilst the excess values do change with the choice of matching criteria, the interpretation of the results is unaffected. 

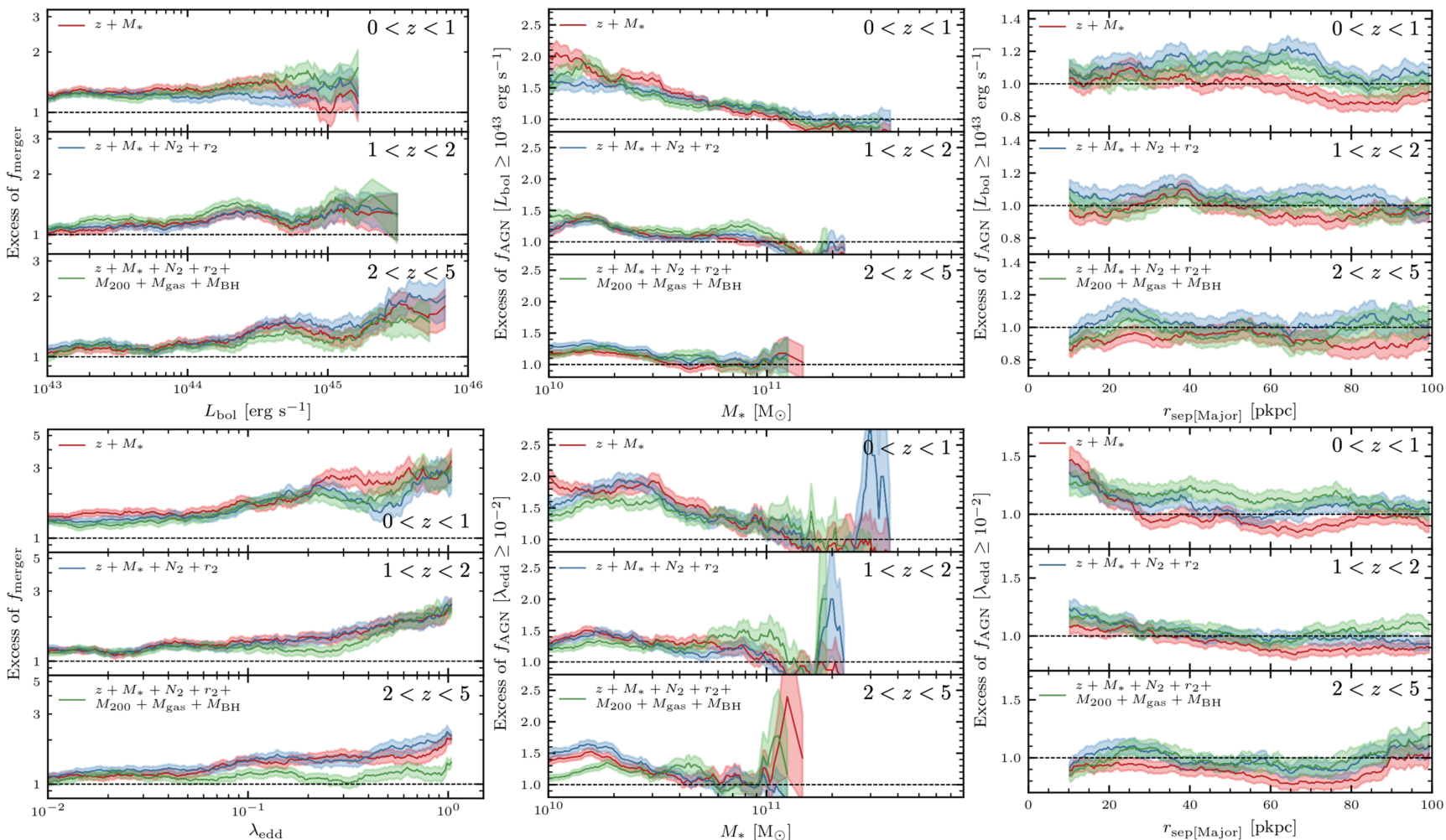

Figure A4. How the excess in the major merger fractions from Fig. 1 (left two panels), the excess in the AGN fractions from Fig. 2 (middle two panels), and the excess in the AGN fractions from Fig. 3 (right two panels) change depending on the control galaxy matching criteria that is used. The legend shows what properties are matched between the selected galaxies and their associated control galaxies. Broadly speaking, the values of the excess are higher when fewer parameters are matched. However, whilst the values of the excess can change depending on the matching criteria used, the overall behaviour in each panel is largely unaffected by the choice of matching criteria.

This paper has been typeset from a $\mathrm{T}_{\mathrm{E}} \mathrm{X} / \mathrm{LA} \mathrm{T} \mathrm{E}$ file prepared by the author. 